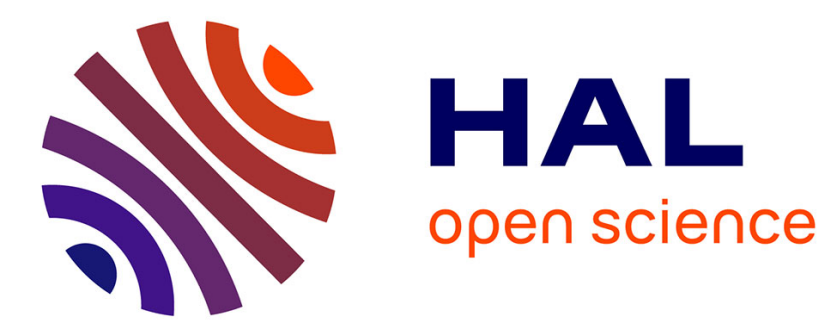

\title{
Optical developments for optogenetics
}

Eirini Papagiakoumou

\section{To cite this version:}

Eirini Papagiakoumou. Optical developments for optogenetics. Biology of the Cell, 2013, 105 (10), pp.443-464. 10.1111/boc.201200087 . hal-02445535

\section{HAL Id: hal-02445535 \\ https://hal.science/hal-02445535}

Submitted on 20 Jan 2020

HAL is a multi-disciplinary open access archive for the deposit and dissemination of scientific research documents, whether they are published or not. The documents may come from teaching and research institutions in France or abroad, or from public or private research centers.
L'archive ouverte pluridisciplinaire HAL, est destinée au dépôt et à la diffusion de documents scientifiques de niveau recherche, publiés ou non, émanant des établissements d'enseignement et de recherche français ou étrangers, des laboratoires publics ou privés. 


\section{Optical developments for optogenetics}

\section{Eirini Papagiakoumou}

Wavefront-Engineering Microscopy Group, Neurophysiology and New Microscopies Laboratory, CNRS UMR 8154, Inserm S603, Paris Descartes University, 45 rue des Saints-Pères, 75270 Paris Cedex 06, France

email: eirini.papagiakoumou@parisdescartes.fr

tel : +33 142864371

fax : +33 142864255

keywords : optogenetics, illumination methods, digital holography, spatio-temporal focusing, generalized phase contrast 


\section{Abstract}

Brain intricacies and the difficulty that scientists encounter in revealing its function with standard approaches such as electrical stimulation of neurons have led to the exploration of new tools that enable the study of neural circuits in a remote and non-invasive way. To this end, optogenetics has initialized a revolution for neuroscience in the last decade by enabling simultaneous monitoring and stimulation of specific neuronal populations in intact brain preparations through genetically targeted expression of light sensitive proteins and molecular photoswitches. In addition to ongoing molecular probe development and optimization, novel optical techniques hold immense potential to amplify and diversify the utility of optogenetic methods. Importantly, by improving the spatio-temporal resolution of light stimulation, neural circuits can be photoactivated in patterns mimicking endogenous physiological processes. The following synopsis addresses the possibilities and limitations of optical stimulation methods applied to and developed for activation of neuronal optogenetic tools.

\section{Introduction}

The attempts to explore the function of the nervous system dates back more than 200 years to Luigi Galvani's (1737-1798) famous experiments in the late $18^{\text {th }}$ century. By causing a frog's legs to twitch by connecting the lumbar nerve to an electrical current source (Piccolino, 2006), Galvani revealed the first and perhaps most fundamental principle of the neural code: that information is transmitted in the form of electrical impulses. Galvani's approach of probing the nervous system with electrodes remains the principle mode of neural circuit investigation today due to high signal-to-noise (S/N) ratios and temporal resolution, as exemplified, for example, by patch clamp recording (Hamill et al., 1981).

Electrophysiology suffers, however, from fundamental limitations. Implanting electrodes into the brain is invasive and difficult to accomplish in freely moving animals. Furthermore, there is a physical limit to the number of electrodes that can be simultaneously inserted into brain tissue. Hence, intracellular patch-clamp recordings are limited to a small number of cells, compared to population size of most cerebral circuits (Ko et al., 2011; Watt et al., 2009). Although patchclamp of principal dendrites or axons has been demonstrated (Stuart and Sakmann, 1994; Stuart et al., 1997), access to small compartments such as dendritic branches and spines continues to prohibit direct electrical recording. Alternately, extracellular electrodes permit simultaneous access to several cells and populations (Buzsáki, 2004) but at the cost of spatial resolution.

The first and primary application of light to cellular biology was for imaging. In the 1980s, it was exploited for trapping and manipulating of micro-particles and cells (Ashkin and Dziedzic, 1987; Ashkin et al., 1986) and its use for investigation of cellular structure and function has exponentially grown in the 1990s with the discovery of genetically targetable fluorescent 
proteins (Heim et al., 1994, 1995). Over the past decade, light has become an 'active' tool for neuronal stimulation. The idea of using light as an alternative way to stimulate neurons is not new, however, dating back to 1971 when Richard Fork used laser light to stimulate nerve cells (Fork, 1971). Although Fork's approach was not widely adopted at that time, probably because the cellular response he got was considered a result of side effects, today a growing number of researchers choose light in place of electrodes, as a less invasive wireless form of activation (Scanziani and Hausser, 2009). Moreover, light enables communication with many receivers in parallel with spatial and temporal resolution adequate to follow the dynamics of physiological processes. Since 1971, another work reporting laser irradiation of neurons was published in 1983 by Farber and Grinvald (Farber and Grinvald, 1983), who used laser excitation of leech neurons stained with a fluorescent dye to evoke action potentials (APs) in the cells. In the 1990s the development of caged glutamate enabled indirect photostimulation of neurons through photolysis of glutamate with flash lamps or lasers (Callaway and Katz, 1993; Katz and Dalva, 1994). Later, pulsed femtosecond lasers were utilized for neuronal stimulation through partial disruption of cell membranes (Hirase et al., 2002).

The real revolution, however, came when studies on rendering neurons actively responding to light started coming up. The laboratories of Gero Miesenböck, while at Memorial SloanKettering Cancer Center, and of Ehud Isacoff, Richard H. Kramer and Dirk Trauner, then all at the University of California, Berkeley, employed methods to genetically engineer and target proteins that directly alter membrane conductivity upon light illumination of specific wavelength (Banghart et al., 2004; Zemelman et al., 2002). The revolution continued with functional expression of natural photosensitive proteins that can be used as cell actuators, such as channelrhodopsin (Nagel et al., 2003), and light-activated G-protein-coupled receptors (GPCRs; Levitz et al., 2013; Panda et al., 2005; Qiu et al., 2005), as well as genetically encoded fluorescent proteins, both calcium-based (Nakai et al., 2001) and voltage-based (Dimitrov et al., 2007; Siegel and Isacoff, 1997), that can be used as functional reporters of neuronal activity. Continued efforts to improve the performance of these molecular innovations for study of neuronal function and treatment of diseases and disorders have coalesced to form the field of optogenetics (Deisseroth et al., 2006; Miesenböck, 2009).

Excitation of the cells expressing such photosensitive compounds is most commonly accomplished by illuminating the sample with a common epi-fluorescence lamp or a lightemitting diode (LED). This illumination method is simple to implement and easily adaptable to in vivo applications through an optical fiber. Its lack of specificity has been counterweighed by targeting photosensitive proteins to specific neuronal subpopulations with specific gene promoters. Thus, although the light is incident across a large field, only targeted neurons are excited. The majority of current studies utilize channelrhodopsin-2 (ChR2), a cation channel highly sensitive to visible light. In ChR2-expressing neurons APs can be evoked with excitation 
powers of $\sim 1 \mathrm{~mW} / \mathrm{mm}^{2}$ at the maximal absorption wavelength of $470 \mathrm{~nm}$, and thus wide-field illumination with blue light provides effective excitation. Indeed, the high sensitivity of ChR2 has been critical for its successful utilization especially for in vivo studies (Aravanis et al., 2007; Gradinaru et al., 2007).

Still, elaborated photosensitive tools for neuronal stimulation require elaborated methods to exploit their full potential. This is something that was understood from the beginning; Richard Fork finishes his article on neuronal photoactivation with the following phrase: "Attempts are being made to improve this technique by limiting the area of stimulation still further." To individuate subsets of genetically identical connected cells, for example, or establish the role of specific spatiotemporal excitatory patterns in guiding animal behavior, innovative optical techniques are needed, able to modulate stimulation for reproducing physiological patterns of neuronal activation. This is, however, a challenging technical issue due to the complexity of neuronal systems in terms of spatiotemporal precision.

The following review details optical methods used so far for optogenetics as well as recent technical developments and their potential applications. The techniques presented here will be discussed specifically in the context of optogenetic applications, but in principle can be extended to any application demanding spatially and temporally precise light stimulation, such as photolysis of caged neurotransmitters, morphological and functional imaging.

\section{Wide-field illumination approaches}

Wide-field illumination is the first method used to stimulate optogenetic tools, as mentioned above, due to its simplicity, requiring essentially inexpensive, off-the-shelf components, such as a fluorescence lamp coupled to a fast shutter. In fact, the first photoactivation experiment of sensitized vertebrate neurons to light was done with white light illumination (Zemelman et al., 2002). Epi-fluorescent activation has been used for a first characterization of optogenetic actuators (Banghart et al., 2004; Kleinlogel et al., 2011; Zemelman et al., 2003; Zhang et al., 2007). It has been also used in combination with fast CCD cameras for recording signals from voltage-sensitive fluorescent proteins (VSFPs), since in that case it facilitates collection of photons from large areas, essential for VSFPs where the collected fluorescence signal is very small (Akemann et al., 2010; Chanda et al., 2005; Dimitrov et al., 2007). In addressing more specific biological questions epi-fluorescent activation of ChR2 at 460-470 nm was used e.g. in mechanosensory neurons of the nematode Caenorhabditis elegans (C.elegans) to trigger specific behaviors (Nagel et al., 2005), to map circuits between presynaptic ChR2-positive neurons and postsynaptic neurons (Petreanu et al., 2007), to investigate active synaptic contacts in rat hippocampal slice cultures (Zhang and Oertner, 2007) and long term potentiation in combination with two-photon (2P) uncaging (Zhang et al., 2008). Wide-field excitation of ChR2 was also used to study $\mathrm{Ca}^{2+}$ elevation in astrocytic cultures in comparison to other 
optogenetic actuators, like the $\mathrm{Ca}^{2+}$-translocating channelrhodopsin (CatCh; Kleinlogel et al., 2011) and the light-gated $\mathrm{Ca}^{2+}$-permeable ionotropic glutamate receptor (LiGluR; Volgraf et al., 2006) (Li et al., 2012), or to examine the function of dopamine in the olfactory bulb of adult zebrafish (Bundschuh et al., 2012).

In some of these studies photoactivation is combined with calcium imaging for monitoring the neuronal activity. Combining photostimulation with calcium or voltage imaging necessitates sufficiently distinct excitation spectra for the photoexcitable neuronal actuator and the functional reporter in order to avoid light-driven neuronal excitation during imaging and the inverse. Indeed, dyes with excitation spectra overlaping little with common optogenetic tools, like ChR2, are commercially available (Zhang et al., 2006). An alternative solution to this problem is to perform imaging with optical methods that provide better spatial resolution and less probability to excite both the functional reporters and the actuators. In the studies described above, for example, 2P calcium imaging was used to excite the calcium indicators Fluo-5F (Zhang and Oertner, 2007) or Rhod-2-AM (Bundschuh et al., 2012), while nearmembrane calcium imaging with evanescent-field excitation (Axelrod, 1981) of Fluo-4 or Xrhod1 was performed to monitor the calcium responses in astrocytes (Li et al., 2012). Recently, a family of red-fluorescent calcium indicators has been presented (Collot et al., 2012) with longer excitation and emission wavelengths, suitable for simultaneous $2 \mathrm{P}$ calcium imaging and excitation of optogenetic actuators.

In-vivo applications demand further adaptation of optogenetics instrumentation, even for simple wide-field excitation. For in vivo light delivery in freely moving animals, lasers or LEDs of sufficient power are coupled to optical fibers ( $10-15 \mathrm{~mW}$ are required at the output of a fiber of $100 \mu \mathrm{m}$ in diameter) and attached to the animal's skull with stereotactic surgery. The diameter of the fiber core (without plastic cladding) depends on the species and the experiment. For mice, up to $300 \mu \mathrm{m}$ fibers can be used without compromising animal movement, whereas rats can tolerate up to $400 \mu \mathrm{m}$ fibers (Zhang et al., 2010). Commercial vendors offer the lasers pre-coupled to optical fibers. To target deep brain structures, thin optical fibers can be used to efficiently transmit sufficient powers of light to the target area (Adamantidis et al., 2007; Aravanis et al., 2007). Furthermore, fiberoptics fused to recording electrodes (optrodes) have also been developed for simultaneous electrophysiological recording and photostimulation of genetically expressed photosensitive ion channels and pumps (Anikeeva et al., 2012; Gradinaru et al., 2007; Wang et al., 2012; Zhang et al., 2009) and GPCRs (Gutierrez et al., 2011) in freely moving mice. For superficial stimulation of cortical layers, miniaturized LEDs can also be directly mounted above the brain over a thinned skull area or cranial glass window (Gradinaru et al., 2007; Huber et al., 2008). 
Simple wide-field illumination of optogenetic probes, despite its inherent lack of spatial specificity, has nonetheless generated important insights into neuronal function and dysfunction. For instance, by combining optogenetics with functional magnetic resonance imaging ( $\mathrm{fMRI}$ ) investigators verified that the firing of local excitatory neurons is sufficient to trigger downstream hemodynamic phenomena detected by fMRI scanners (Lee et al., 2010) or to facilitate appropriate plasticity by guiding the cortical reorganization after nerve injury (Li et al., 2011). In addition, photostimulation of the light-activated chloride pump halorhodopsin from Natronomonas pharaonis (eNpHR) (Zhang et al., 2007) or of the $\mathrm{K}^{+}$-channel small molecule photoswitch AAQ (acrylamide-azobenzene-quaternary ammonium; Fortin et al., 2008) allows restoration of visual responses in mice and in human ex vivo retinas (Busskamp et al., 2010; Polosukhina et al., 2012). ChR2 activation was used to control heart muscle both in vitro and in vivo (Bruegmann et al., 2010), and breathing in anesthetized mice (Depuy et al., 2011). Optogenetic stimulation also shows promise for treating mental diseases, already used to reveal that cholinergic interneurons can block cocaine conditioning (Witten et al., 2010) and to activate fear memory recall (Liu et al., 2012). Moreover, optogenetic tools have been used to treat sleeping disorders by stimulating hypocretin neurons implicated in narcolepsy (Adamantidis et al., 2007), to stimulate dopamine neurons in order to understand their function in depression and substance abuse (Tsai et al., 2009), to acquire fundamental knowledge for Parkinson's disease and the mechanisms of action of therapeutic interventions (Kravitz et al., 2010) and to control the activity of neocortical parvalbumin interneurons, implicated to mental dysfunctions characteristic of schizophrenia or autism (Cardin et al., 2009; Sohal et al., 2009).

In combination with the high sensitivity to visible wavelengths, the above examples were made possible through a remarkable development of gene delivery methods including viral expression, transgenic mice, in utero electroporation (see Zhang et al., 2010 for a complete description of genetic strategies to target specific neuronal populations). Both the resounding success and limitations of early optogenetic studies manifested the need for more flexible and specific illumination tools to differentially activate cells of the same population or to target subcellular structures. Thus, the development of optical techniques that offer higher spatial and temporal resolution has become a necessity, especially for efforts to combine photoactivation with fluorescent functional reporters where the use of wide-field excitation often reduces the $\mathrm{S} / \mathrm{N}$ ratio, due to out-of-focus fluorescence signal.

\section{Patterned visible excitation}

Light patterning techniques enable fast, remote modification of the spatial configuration of light delivered to tissue, without the need to intervene on the optical setup during the experiment. Such techniques can be useful for stimulating a subpopulation of genetically identical neurons or targeting subcellular structures, like dendrites, axons or dendritic spines. Several schemes 
have been proposed to perform spatial shaping of the excitation beam, generally classified in two categories: scanning methods, in which serial excitation of several regions of interest (ROIs) is performed by rapidly scanning the laser focus, and parallel methods, in which all ROls are simultaneously excited.

\section{Scanning methods}

The easiest way to create pattern-like excitation is by scanning a focused laser beam (Katz and Dalva, 1994). This approach has been used to map synaptic connectivity by photostimulating ChR2 with blue laser radiation focused to a small spot $(\sim 1 \mu \mathrm{m})$ and scanned over different locations to assess light sensitivity (Wang et al., 2007a). Other investigators focused the laser to a near cylindrical beam of 6-16 $\mu \mathrm{m}$ full width at half-maximum (FWHM), scanned over the dendrites of the recorded neuron (Petreanu et al., 2009). Single-cell scanning excitation of ChR2 was also recently used to map network connections between two distinct cortical inhibitory networks of the visual cortex in vivo (Wilson et al., 2012). In these examples the position of the laser beam was changed by rapidly deflecting its direction with a pair of galvanometer-driven mirrors that dictated the position of the spot in $\mathrm{x}$ and $\mathrm{y}$. Such scanning systems are now commercially available and can be easily adapted to standard microscopes by imaging the scanner with a telescope to the back aperture of the objective (Figure 1A). The main advantage of scanning the beam with mirrors is that there are no significant light losses in the optical path. However, scan speed is limited by mechanical inertia and by the dwell time necessary to generate a sufficient physiological response (scanning frequencies of few $\mathrm{kHz}$ have been reported; Gasparini and Magee, 2006). Thus, although scanning has been successfully applied to excite single cells, the scan speed limitation has hindered application of this method to neural network questions requiring simultaneous stimulation of multiple neurons.

Scan speed limitations can be addressed in part by using resonant scanning mirrors (Fan et al., 1999; Nguyen et al., 2001) or acousto-optic deflectors (AODs) (Reddy et al., 2008) to deflect the beam's path much faster with frequencies reaching tens of $\mathrm{kHz}$. Resonant scanning galvanometer mirrors are mirrors that oscillate in a sinusoid manner. These devices cycle at a resonant frequency on the order of 4 to $8 \mathrm{kHz}$, while bidirectional scanning makes the effective scan rate $\sim 16 \mathrm{kHz}$ (Fan et al., 1999). AODs are devices in which a propagating sound wave of radio frequency through an acousto-optic medium establishes a grating that diffracts a laser beam at a precise angle, which can be modified within few microseconds by changing the frequency of the sound wave. When a monochromatic light beam traverses an AOD, a large fraction of light is 'deflected' at an angle, $\theta$, that is linearly related to the sound frequency, $f$ : $\theta=\lambda f / v$, where $\lambda$ is the wavelength of the incident light and $u$ is the velocity of the propagating sound wave. This effect can be used for highly versatile inertia-free scanning schemes, because the deflection angle is controlled by electronically generated frequencies without moving any mass-containing elements (i.e. mirrors). Consequently, a two-AOD scanning 
system enables two-dimensional (2D) patterns in discontinuous scan modes, and more complex four-AOD configurations with counter-propagating chirped acoustic waves enable in addition axial scanning of the laser beam (Reddy and Saggau, 2005; Reddy et al., 2008). However, the excitation field that can be achieved with AODs is small comparing with scanning mirrors, (typical values 150×150 - 200×200 $\mathrm{m}^{2}$; Shoham et al., 2005; Reddy et al., 2008) depending on the available range of acoustic frequencies, which defines the maximum beam deflection angle. Moreover, since AODs are diffracting elements, they have a certain diffraction efficiency (ratio of light that goes to the first diffraction order relatively to the total amount of light entering the AODs) that ranges between $50 \%$ and $70 \%$ for a two-AOD scanning system (Losavio et al., 2009; Shoham et al., 2005). Recently, new design concepts that improve the performance of a 4-AOD system have been presented, with the possibility to image neuronal somata within a volume of $700 \times 700 \times 1400 \mu \mathrm{m}^{3}$ and dendrites within a volume of $290 \times 290 \times 200 \mu \mathrm{m}^{3}$ (Katona et al., 2012).

Resonant scanners have been used mainly for 2P calcium imaging (Fan et al., 1999; Nguyen et al., 2001; Rochefort et al., 2009), while AOD scanners have been widely used in 2D for uncaging applications (Losavio et al., 2009; Shoham et al., 2005) and 2P calcium imaging (Otsu et al., 2008; Salomé et al., 2006) including also three dimensions (3D) (Katona et al., 2012; Reddy and Saggau, 2005; Reddy et al., 2008). In addition, investigators recently used AODs to photostimulate optogenetic actuators (Wang et al., 2011), specifically manipulating the activity of neurons expressing the ameliorated chimera variant of channelrhodopsin ChIEF (Lin et al., 2009), achieving high temporal resolution with single-photon excitation (1PE).

Scanning methods, even those using resonant scanners or AODs to achieve fast scan rates, remain serial approaches limited by the dwell time, a parameter independent of how fast the beam is scanned from one site to another. Exciting one or two cells (Wang et al., 2011) in a 'simultaneous' manner may be possible by the long opening time of opsins (Lin et al., 2009). However, since temporal resolution of scanning methods, $T_{S}$, is determined by the sum of the scan time $\left(t_{s}\right)$ and the dwell time $\left(t_{d w e l l}\right)$ for each visited position: $T_{S}=N\left(t_{s}+t_{d w e l l}\right)$, where $\mathrm{N}$ is the number of visited sites, the number of cells that can be serially accessed for nearsimultaneous stimulation is quickly limited.

\section{Parallel methods}

Parallel excitation methods arose as a solution for the 'simultaneity' problem. These methods utilize spatial light modulators (SLMs) to change the intensity profile of the laser beam. In this way 2D or 3D distributions of multiple diffraction-limited spots and arbitrary excitation patterns mimicking cellular morphology can be realized at the sample plane. Hence, parallel methods enable high spatial flexibility, since illumination patterns can be easily adapted to different experimental requirements within the same optical configuration. The beam incident on the SLM can be described as an electromagnetic wave of a certain amplitude, $A$, and spatial phase, 
$\Phi: \varepsilon=A \cdot e^{i \Phi}$, where $i$ is the imaginary unit. Thus, beam modulation can be achieved either by modulating the amplitude or the phase with the SLM.

\section{Amplitude modulation}

Amplitude modulation is performed by directly shaping the intensity of light in a plane conjugated to the sample plane (Figure 1B). The SLMs most commonly utilized for this configuration are digital micromirror devices (DMDs), which are arrays of micromirrors (commercially available devices with up to $1024 \times 768$ or $1920 \times 1080$ individual micromirrors) suspended on air gap and controlled electrostatically. Each mirror can be rotated along its diagonal by $\pm 12^{\circ}$ from the unpowered position via a control signal (Knapczyk and Krishnan, 2005). In this way light can be independently deflected by each mirror creating spatially extended arbitrary patterns. DMDs have been used widely for optogenetics in cell cultures (Wang et al., 2007b), to control neuronal activity patterns in zebrafish (Arrenberg et al., 2010; Wyart et al., 2009; Zhu et al., 2012) and in C.elegans (Guo et al., 2009; Leifer et al., 2011), and to probe odor coding mechanisms in vivo in the olfactory bulb (Dhawale et al., 2010). As an alternative to DMDs, digital amplitude modulation with a modified off-the-shelf liquid crystal display (LCD) projector has been also presented (Stirman et al., 2011, 2012).

Works where high power micro-LED arrays in a 1:1 imaging configuration or demagnified at the sample plane for excitation of dendrites were used to excite ChR2-expressing neurons in vitro (Degenaar et al., 2009; Grossman et al., 2010) have been presented, as well. However, as microLEDs have fewer pixels than DMDs (64x64), a serious compromise between the spatial resolution and the excitation field must be made in that case. For example, in an excitation field of $3 \times 3 \mathrm{~mm}^{2}$ a single light spot is $30 \mu \mathrm{m}$ FWHM, while reducing the size of the light spot to $3 \mu \mathrm{m}$ FWHM limits the excitation field to $0.3 \times 0.3 \mathrm{~mm}^{2}$ (Grossman et al., 2010).

To tightly couple optical stimulation with 2D electrical recording over multiple sites for in vivo studies in mice and rats, tapered optical fibers were glued to commercially available 4- or 8electrode linear arrays (Royer et al., 2010). These 2D electrical-optical arrays were used for stimulation and parallel recording of ChR2-transfected rat hippocampal neurons. Other investigators extended this approach for 2D control of light delivery with microfabricated multiwaveguide probes (Zorzos et al., 2010) or probes with integrated micrometer-sized LEDs (McAlinden et al., 2013).

The greatest advantage of amplitude modulation methods is the high temporal and, in many cases, high lateral resolution. DMDs, for example, can update intensity patterns at rates of 1-10 kHz (Wilt et al., 2009) and micro-LEDs can switch on a nanosecond time scale (Grossman et al., 2010), thus easily mimicking rapid physiological processes. The limit on time resolution for amplitude modulation methods is in fact the activation/deactivation kinetics of photoactive compounds, the primary determinant of dwell time. 
The Achilles' heel of amplitude modulation, however, is the large fraction of light power lost, since the pattern is created by redirecting unwanted light out of the excitation field in the case of DMDs or by switching off the corresponding emitters in micro-LEDs. For this reason amplitude modulation techniques are primarily utilized for single-photon excitation of optogenetic actuators, for which demands in terms of power are modest compared with twophoton.

\section{Phase modulation}

For light-patterning through phase modulation, the phase wavefront of the laser beam is modulated at the back aperture of the microscope objective such that the desired excitation pattern is generated in focus at the sample plane. Phase modulation is most commonly performed with liquid crystal (LC)-based SLMs, pixelated liquid crystal matrices that modulate the spatial phase of an incident wavefront in a full range of $[0,2 \pi]$ by controlling the refractive index of each pixel. Refractive index changes are induced by re-orienting liquid crystal molecules with an electric field. The SLM typically occupies a plane conjugated with the back focal plane of the objective (Figure 1C). A variety of algorithms exist for calculation of phase modulation maps addressed to the SLM (also called computer-generated holograms, CGHs), the choice of which depends on the application. For example, iterative Fourier-transform algorithms (Gerchberg and Saxton, 1972) are used to generate extended excitation patterns, since objective lens focusing can be simulated by a mathematical Fourier transform. For the generation of multiple diffraction-limited spots in independent axial planes, a similar more efficient iterative algorithm improves the intensity uniformity among the spots (Di Leonardo et al., 2007), however simpler algorithms enabling control of spots' lateral position with a grating phase effect and spots' axial position with a lens phase effect can also be used (Liesener et al., 2000).

Computer generated hologram laser beam phase modulation, here forward referred to as digital holography (DH), was first utilized for generation of multiple optical traps with optical tweezers (Curtis et al., 2002; Reicherter et al., 1999). Application of DH for neuronal photoactivation was first demonstrated for photolysis applications (Lutz et al., 2008; Nikolenko et al., 2008). Although DH found broad application for 1P uncaging (Anselmi et al., 2011; Santos et al., 2012; Yang et al., 2011; Zahid et al., 2010), its utility for 1PE of optogenetic tools was only recently demonstrated in stimulation of ChR2-expressing retinal ganglion cells of blinded retinas for bionic vision restoration (Reutsky-Gefen et al., 2013). Figure 2 illustrates another example of 1P holographic excitation of ChR2-expressing HEK cells and cultured neurons. Specifically, figure 2A shows holographic excitation extended over an entire cell's surface, demonstrating also DH's fine spatial specificity as patched cells register no response when patterned light is delivered to neighboring cells. Figure $2 \mathrm{~B}$ illustrates modulation of the induced photocurrent by exciting the whole soma (black trace) or the principal dendrite (red trace) of the recorded neuron. 
An advantage of phase over amplitude modulation is that power losses are minimized by redistributing, rather than deviating or blocking, light at the sample plane for formation of the desired intensity profile. Furthermore, DH offers an improved axial resolution for extended spots in comparison with large Gaussian beams (Lutz et al., 2008; Vaziri and Emiliani, 2012; Zahid et al., 2010), arising from the ability to generate large excitation areas by overfilling the objective back aperture, exploiting its full focusing strength. In contrast, large area excitation with Gaussian beams necessitates underfilling the objective back aperture at cost of the axial confinement of the excitation volume. Moreover, since DH is a scan-less method, the temporal resolution of photoactivation is imposed only by the dwell time necessitated by optogenetic actuator kinetics: $T_{D H}=t_{d w e l l}$.

DH suffers two primary drawbacks: light lost to diffraction at the SLM and intensity inhomogeneities caused by speckle. The spatially varying diffraction efficiency of LC-SLMs, causes light loss in the formation of zero- and higher diffraction orders, limiting the excitation field (Arrizón and Testorf, 1997; Palima and Daria, 2006). Light sent to zero- or higher diffraction orders can be minimized by using liquid crystal on silicon (LCOS) SLMs and, in addition, suppressed through adaptation of the original phase hologram (Jesacher and Booth, 2010; Palima and Daria, 2007; Ronzitti et al., 2012). The excitation field size for DH depends on the SLM pixel size, $\alpha$, and its demagnification at the sample plane (Golan et al., 2009; Yang et al., 2011). In the lateral direction, it can be calculated by the following equation: $\Delta \mathrm{X}_{\max }=\Delta \mathrm{Y}_{\max }=$ $2 \frac{\lambda \cdot f_{o b j}}{2 \alpha} \frac{f_{1}}{f_{2}}$, where $\lambda$ is the illumination wavelength, $f_{o b j}, f_{1}$ and $f_{2}$ are the focal lengths of the objective and the lenses used to demagnify the SLM array to the back focal plane of the objective (Figure 1C). The excitation field is typically defined as the area in which the intensity of the desired laser pattern reaches at least $50 \%$ of the total intensity incident on the SLM, corresponding to a diffraction efficiency $\geq 50 \%$. For example, for a $40 x, 0.8 \mathrm{NA}$ objective, frequently used for biological applications, and $\frac{f_{1}}{f_{2}}=1.5$, the excitation field is roughly $100 \times 100$ $\mu \mathrm{m}^{2}$, much smaller than the excitation field achievable with scanning methods ( $\sim 600 \times 600 \mu \mathrm{m}^{2}$ for galvanometer-driven mirror based systems; Oron et al., 2012). The field size limitation is the primary reason that limits the use of $\mathrm{DH}$ to photoactivation applications, and it is not preferred for imaging.

Speckle-induced intensity inhomogeneities within the desired light pattern pose the second primary disadvantage for $\mathrm{DH}$. Speckles arise due to interference of light caused by diffraction through adjacent finite-aperture pixels and the fact that the iterative algorithm modulates only the phase of the laser beam (Palima and Glückstad, 2008). Hence, when calculating the final solution for the electromagnetic field in each iteration, the amplitude is ignored and replaced by the Gaussian beam incident onto the SLM (Oron et al., 2012), leading to an approximate solution in which holographic patterns exhibit a speckled intensity profile (Figure 2). These 
fluctuations can reach $20 \%$ in $1 \mathrm{PE}$ and $50 \%$ in $2 \mathrm{P}$ excitation (2PE). Ideally, one should modulate both the phase and the amplitude of the waveform in order to achieve a perfect reconstruction of the desired pattern (Jesacher et al., 2008), but, as previously mentioned, amplitude modulation results in loss of light power. Efforts to eliminate holographic speckle include time averaging either different speckle patterns with a rotating diffuser (Papagiakoumou et al., 2008) or multiple shifted versions of a single hologram (Golan and Shoham, 2009; Matar et al., 2011). In the first case, however, there is a significant loss of light power and in the second case the temporal resolution is limited by the refresh rate of LC-SLMs $(60-200 \mathrm{~Hz})$ for shifting between different holograms. Ferroelectric liquid crystal SLMs, capable of $>1 \mathrm{kHz}$ refresh rates, were used in the above references to improve the temporal resolution of shifting the $\mathrm{CGH}$. However, these devices can perform only binary $[0, \pi]$ phase modulation, resulting in low diffraction efficiency ( $40 \%$ ), consequently reducing the excitation field (Golan et al., 2009). Although supplying sufficient light power for 1PE in optogenetics (Reutsky-Gefen et al., 2013), significant losses to diffraction preclude implementation of this solution for applications demanding more power, such as $2 \mathrm{PE}$.

A different approach to project complex field holograms, using a phase to intensity mapping technique, has been recently presented (Go et al., 2011). Investigators used the generalized phase contrast method (see the 'Phase modulation' section in the 'Two-photon excitation' paragraph) for amplitude modulation in combination with $\mathrm{DH}$ for phase modulation, demonstrating efficient generation of near diffraction-limited spots. Although not shown, the projection of extended light patterns should be also feasible.

\section{Two-photon excitation for optogenetics}

Although the above-described methods enabled significant gains in temporal resolution and lateral spatial patterning for actuation of optogenetic tools with visible wavelengths, limitations inherent to $1 \mathrm{PE}$ systems, i.e. scattering and lack of axial optical sectioning, prevent single cell targeting and limit excitation to shallow depths. Two-photon (or in general multi-photon) fluorescence excitation remedies both limitations (Denk et al., 1990). For 2PE, the probability of observing quasi-simultaneous absorption of two-photons by a molecule falls off with the intensity squared, conferring optical sectioning. In addition, the longer, near-infrared wavelengths used for 2PE are less scattered than visible wavelengths, enabling excitation hundreds of microns deep in scattering tissue (Helmchen and Denk, 2005).

In optogenetics 2PE is widely used for imaging genetically encoded functional reporters (see 'Excitation by scanning approaches'). However, 2PE of optogenetic actuators is not as trivial as for $1 \mathrm{PE}$, even with the large $2 \mathrm{P}$ absorption cross-sections demonstrated for probes like ChR2 (260 Goeppert-Mayer at $920 \mathrm{~nm}$ empirically estimated by Rickgauer and Tank, 2009). Although some evidence incriminates poor infrared absorption by the all-trans-retinal, the light-sensitive 
element of ChR2, rather than the features of the protein itself (Kasparov and Herlitze, 2013), there are also other properties of these channels hindering their use in high spatial resolution 2PE schemes. First, most opsins, including ChR2, have a small single-channel conductance ( 80 fS; Feldbauer et al., 2009) much lower than the one of endogenous channels. Thus, the photocurrent generated by the few channels occupying the femtoliter volume of a $2 \mathrm{P}$ diffractionlimited spot is too small to induce sufficient membrane depolarization for the generation of APs. This limitation is especially salient for channels such as ChR2, for which low conductance cannot be compensated by increasing the intensity and duration of the illumination spot, due to the long lifetime of the excited conducting state. Prolonged illumination with high light intensity results in substantial depletion of the ground state, with further increase yielding a saturated response (Rickgauer and Tank, 2009). Furthermore, at high light intensities out-of-focus excitation can induce unwanted photocurrents in other cellular compartments or neighboring cells that can easily exceed currents excited in the small focal volume, thus offsetting any gains in spatial specificity. Out of focus excitation can be avoided by scanning the laser spot over the membrane of a target neuron at a low intensity still sufficient to excite most available current in-focus. However, the fast ( millisecond) deactivation time of ChR2 prevents the adequate temporal summation of currents generated through serial scanning. $2 \mathrm{P}$ activation of ChR2 was reported for the first time in 2008 (Mohanty et al., 2008) by recording neuronal responses in hippocampal brain slices with $2 \mathrm{P}$ calcium imaging. However, the small fluorescence reported by the calcium dye indicated small amplitude, sub-threshold activation. Hence, the methods for efficient yet spatially confined 2PE of ChR2 and other actuators needed to be reconsidered.

\section{Excitation by scanning approaches}

The first paper reporting rigorous 2PE of ChR2 in cultured neurons used a laser-scanning approach with galvanometer-driven mirrors (Rickgauer and Tank, 2009). To improve excitation efficiency, laser spot size was increased by decreasing the effective numerical aperture (NA) of the objective with an iris placed near the back focal plane, and the spot was scanned over a spiral trajectory to cover the whole cell surface. With an effective NA of 0.3 (instead of the objective's nominal NA 0.8) and overall scanning time of 30 ms, Rickgauer and Tank demonstrated the first 2PE AP generation in ChR2-expressing neurons. The obvious disadvantage of this approach is limited temporal resolution, which in principle could be increased by further decreasing the NA, so that the spot focal volume simultaneously opens more channels. However, NA reduction also degrades axial resolution, defined as the 1/e width of the squared intensity profile in $z, \omega_{z}$, described for 2PE by Zipfel et al., 2003.

A similar approach, improving the temporal resolution performance of scanning methods with low-NA excitation beams, was published about a year later (Andrasfalvy et al., 2010). Andrasfalvy et al. recuperated the lost optical confinement for the low-NA excitation spots by temporally focusing the excitation beams. Temporal focusing (TF), a technique originally 
introduced for wide-field 2P microscopy (Oron et al., 2005; Zhu et al., 2005), permits exceptional depth confinement of the light for large excitation areas. As mentioned previously, the 2 PE fluorescence signal, $S_{2 P E}$, is proportional to the square of the peak intensity: $S_{2 P E} \propto$ $I^{2}=\left(\frac{E}{\tau A}\right)^{2}$, where $E$ is the pulse energy, $\tau$ is the pulse duration and $A$ is the surface of the excitation beam. Typical spatial focusing achieves optical sectioning by tightly focusing the laser beam to a very small spot, such that if spot size increases, optical sectioning degrades with decreasing peak intensity. In contrast TF operates through pulse duration modification; that is the pulse is compressed as it propagates through the sample, reaches its shortest duration at the focal plane and stretches again as it propagates beyond. Thus, lost optical sectioning due to the increase of the excitation spot size is regained through increased pulse duration outside of the focal volume.

TF is realized experimentally with a diffraction grating imaged onto the sample via a telescope formed by a regular lens and the objective (Figure $3 \mathrm{~A}$ ). Briefly, the grating disperses frequencies comprising the spectrum of the femtosecond pulse (typically $100 \mathrm{fs}$ pulses for 2PE microscopy; bandwidth $\sim 10 \mathrm{~nm}$ ) towards different angular directions. Dispersed spectral components are then collimated by the first lens and recombined at the focal plane of the objective. Rays corresponding to different spectral components propagate through the imaging system following different optical paths, eventually recombining with exactly the same phase at the objective focal plane, recovering there its original short pulse duration. In all other planes the rays arrive with a relative phase offset, resulting in illumination of this plane for a longer duration (spectral dispersion; Figure $3 \mathrm{~B}$ ). Since the pulse is broadened out of the focal plane, the probability of $2 \mathrm{PE}$ is low, thus restricting excitation to a small volume around the focal plane, in which the pulse duration is short enough to evoke $2 \mathrm{P}$ absorption. It has been shown, both for multiphoton absorption (Oron et al., 2005) and multiphoton generation processes (Oron and Silberberg, 2005), that the axial resolution achieved for wide-field temporally focused excitation is equivalent to the one of multiphoton line-scanning microscopy. For a detailed description of the phenomenon in frequency or in time domain the reader can refer to the review by Oron et al., 2012.

Andrasfalvy et al. (2010) randomly scanned a temporally focused laser spot of $5 \mu \mathrm{m}$ in diameter over the somata and processes of ChR2-expressing hippocampal neurons in slices at saturation powers for ChR2 (460 $\mathrm{mW}$ at the sample plane, i.e. $>23 \mathrm{~mW} / \mu \mathrm{m}^{2}$ ), eliciting APs with 1-6 ms temporal resolution at depths up to $150 \mu \mathrm{m}$. This approach was used to study the cellular and circuit level mechanisms of hippocampal theta oscillations (Losonczy et al., 2010). However, the high excitation power relative to Rickgauer and Tank, employed to trigger APs with high temporal resolution, canceled excitation confinement gained with temporal focusing. Lateral and axial spatial resolution for this approach was deduced by displacing a 5 - $\mu \mathrm{m}$ size spot across 
a thin dendrite and through different axial planes respectively (Figure 4A), evoking significant depolarization more than $10 \mu \mathrm{m}$ away from the dendrite in the lateral direction and more than $40 \mu \mathrm{m}$ out of plane in the axial direction, despite theoretical estimates for axial resolution of the spot itself of the order of the micron.

Development of new opsins with slower closing times, custom expression, and spectral properties adapted for typical raster scanning rates has recently facilitated scanning approaches, without implementation of temporal focusing or elaborate scanning trajectories. Specifically, these advances enabled 2PE of C1V1 variants, red-shifted chimeric opsins comprised of channelrhodopsin-1 (ChR1) and volvox carteri ChR1 (VChR1; Yizhar et al., 2011), by raster scanning a 1- $\mu \mathrm{m}$ size spot within more than $70 \mathrm{~ms}$, total photoactivation time, at 1064 $\mathrm{nm}$ (Packer et al., 2012) and $\sim 1.3 \mu \mathrm{m}$ size spots at $1040 \mathrm{~nm}$ (Prakash et al., 2012) with much shorter photoactivation time (2.3-9.5 ms). The large discrepancy in the total scan time needed to evoke APs is not easily explained given that both investigations used the same approach at similar excitation powers. Prakash et al. demonstrated 2PE of C1V1 at depths up to $250 \mu \mathrm{m}$ in vivo, as well as 2PE of ChR bistable variants (ChR2-C128T or C128A) and the microbial rhodopsin protein archaerhodopsin eArch3.0, an engineered proton pump (Mattis et al., 2012) with kinetics slower than the chloride pump eNpHR3.0, in cultures and brain slices. The $\sim 1.0-\mu \mathrm{m}$ spot size employed in both cases necessitated excessively high excitation power densities to efficiently excite neurons (typical values: $38 \mathrm{~mW} / \mu^{2}{ }^{2}$, Packer et al., 2012; $15-60 \mathrm{~mW} / \mu \mathrm{m}^{2}$ depending on the objective used, Prakash et al., 2012). The high excitation power degraded the realized axial resolution (Figure $4 \mathrm{~B}-\mathrm{C}$ ), theoretically reaching micrometer precision for such a small excitation spot, and increases the risk of photodamage.

Excitation with 2P diffraction-limited spots by using mirror-based scanning systems has also been applied to functional imaging of genetically encoded calcium indicators, such as the widely known GCaMP family (Akerboom et al., 2012; Chen et al., 2012; Dombeck et al., 2010; Mao et al., 2008; Tian et al., 2009), providing enhanced spatial resolution compared to 1P scanning excitation. For voltage indicators, although some progress has been made with line (Fisher et al., 2008; Kuhn et al., 2008) and point scanning (Acker et al., 2011) and recent engineering of dyes more efficient for 2PE (Acker et al., 2011; Yan et al., 2012), photon-counting limitations due to chromophore saturation during short dwell times, characteristic of conventional laser scanning techniques, have hindered 2PE of genetically encoded voltage sensitive proteins (Knöpfel, 2012).

\section{Parallel excitation}

Although parallel, patterned excitation methods show immense promise to address the challenges detailed above for both 2PE imaging and photoactivation, these techniques are currently in their infancy. The delay is driven by two primary causes, the first emerging from the 
high complexity of the optical setup compared with mirror scanning systems, and the second from the reduced available power density. Since laser power is distributed across the excitation pattern, thus simultaneously illuminating larger areas without loss of temporal resolution, the power density is lower than if the laser was focused to a diffraction-limited spot. However, decreased power density is easily compensated, and in fact surpassed, since parallel excitation optimizes the recruitment speed of optogenetic reporter molecules, thus decreasing the excitation power density necessary to photoactivate, detailed as follows.

\section{Amplitude modulation}

The low efficiency of amplitude modulation methods due to light rejection has thus far prevented their use with 2PE. Especially for DMDs, the maximization of diffraction efficiency requires their implementation at a specific angle, which critically depends on the illumination wavelength. Thus, although experiments in two different wavelengths in 1PE have been successful (Sakai et al., 2013; Wang et al., 2007b) the use of DMDs in combination with broadband or tunable light sources is not straightforward.

\section{Phase modulation}

Thus far, DH has been implemented in 2PE both for the generation of multiple near diffractionlimited spots (Daria et al., 2009; Nikolenko et al., 2008) and extended excitation patterns (Dal Maschio et al., 2010; Papagiakoumou et al., 2008). Since large excitation areas suffer the disadvantage of degraded axial confinement, in 2008 we ameliorated the axial resolution by combining $\mathrm{DH}$ with temporal focusing, although the rapidly varying phase of the holographic beam wavefront slightly distorts color recombination at the focal plane of the objective in comparison to smooth wavefronts, like those of Gaussian beams (Figure 3B). With this technique, we generated scan-less, depth resolved excitation in arbitrary illumination configurations, independently of the excited area size (Papagiakoumou et al., 2008, 2009). Indeed, DH has already proven useful for 2P uncaging applications (Dal Maschio et al., 2010; Go et al., 2012; Nikolenko et al., 2008) and furthermore shows potential for fast imaging applications (Papagiakoumou et al., 2009). Recently, a phase-only SLM was coupled to a standard 2P scanning microscope for inertia-free 3D morphological imaging (Dal Maschio et al., 2011), scanning near diffraction-limited spots in 2Ds while holographically changing their axial position.

A similar approach recently has revealed benefits of $\mathrm{DH}$ for optogenetics by simultaneously evoking APs from two C1V1-expressing neurons lying in planes separated axially by $20 \mu \mathrm{m}$. The neurons were actuated by raster scanning of $1-\mu \mathrm{m}$ holographic spots in $2 \mathrm{D}$, one in each plane (power: $30 \mathrm{~mW}$ per spot; Packer et al., 2012). The same experiments would have been impossible with objective scanning approaches commonly utilized for 3D 2P calcium imaging (Göbel et al., 2006). Indeed, objective scanning is too slow for simultaneous stimulation of cells 
in separate planes, even those expressing C1V1 or other optogenetic actuators with kinectics prolonged specifically for scanning systems.

Two-photon DH offers an alternative to single-spot scanning stimulation of optogenetic actuators with extended arbitrarily shaped patterns. Although, this illumination approach has yet to be demonstrated, preliminary experiments in our laboratory confirm its feasibility. Besides, the efficiency of using extended patterns to activate ChR2 in 2P has been validated with a different phase modulation technique, generalized phase contrast (Papagiakoumou et al., 2010).

Generalized phase contrast (GPC; Glückstad, 1996) belongs to the category of the interferometric phase visualization techniques for which the output image is obtained by the interference between a signal and a reference wave, traveling along the same optical axis. The most well-known example of interferometric phase visualization is Zernike's phase contrast method (Zernike, 1955), which represented a breakthrough for medicine and biology by rendering transparent organisms clearly discernible under the microscope through the visualization of small phase perturbations with a Fourier plane phase shifting filter. The GPC method is an extension of Zernike's phase-contrast into the domain of full range $[0,2 \pi]$ of phase variations (Glückstad and Mogensen, 2001). Briefly, a desired target intensity map is converted into a binary $[0, \pi]$ phase map that modifies the input beam wavefront via the LC-SLM. The beam modulated by the LC-SLM is then focused on a patterned phase contrast filter (PCF) plate imposing appropriate phase retardation between the on-axis focused component (nondiffracted light) and the higher-order Fourier components (Figure 5A). Lastly, the interference between these two beams generates the desired target intensity at the focal plane of a second lens (the output plane).

GPC surpasses DH both in terms of speed and output pattern homogeneity. Since for GPC the phase hologram addressed to the SLM is a simple phase map transformation of the desired output intensity, there is no iterative calculation and thus the image projection is fast and direct, transforming a pure phase modulation to an intensity pattern. Hence, GPC generates patterns free of speckle (Figure 5B), 'ghost' images and zero order, since the zero order interferes directly with the signal wave. In addition, GPC is based on binary phase holograms, thus ferroelectric liquid crystal matrices can be used to further improve the temporal resolution of the technique.

Despite the speed and homogeneity of GPC, DH remains more flexible in terms of the variety of excitation configurations that can be generated. GPC, for example, cannot generate spots in different axial planes. Moreover, conditions for maximum interferometric contrast require a factor of 1:4 ratio between the number of SLM pixels modulated with phase at $\pi$ (corresponding to bright regions in the final intensity map) and the total number of pixels (Palima and 
Glückstad, 2008; Papagiakoumou et al., 2010), thus restricting the size of the excitation pattern area. GPC patterns also lack axial spatial confinement, since out-of-focus planes contain an interference pattern inherent to the interferometric method (for a comparison between the axial propagation of 2P holographic and GPC patterns see Oron et al., 2012). Finally, the GPC excitation field can be adjusted by selecting appropriate telescope lenses for imaging the output plane to the sample plane, as in $\mathrm{DH}$. That is, for a circular aperture in front of the SLM of radius

$R_{C}$, the excitation field, $\Delta R$, will be $\Delta R=\sqrt{\Delta X^{2}+\Delta Y^{2}}=R_{C} \frac{f_{2}}{f_{1}} \frac{f_{o b j}}{f}$, where $f, f_{1}, f_{2}$, $f_{o b j}$ are the focal lengths of the lenses used (Figure 5C). However, for 2P photoactivation, power limitations practically restrict the excitation field to tens of microns for a 40x, 0.8 NA objective.

We implemented GPC for 2PE of ChR2-expressing layer V pyramidal neurons (Papagiakoumou et al., 2010). In order to mitigate limitations imposed by GPC's fill factor requirement, a peripheral ring was sent to the SLM, its weight adjusted for each excitation pattern to maintain the 1:4 ratio. Light sent to the ring was blocked near the GPC output plane. Although the maximum pattern area remained ultimately limited, we were able to excite up to five neurons simultaneously within a circular $60 \mu \mathrm{m}$ diameter excitation field. In addition, the issue of the axial confinement was addressed by combining GPC with TF, imaging the GPC output plane on the diffraction grating used for TF (Figure 5C).

Temporally focused GPC (TF-GPC) patterns mapped over the somatic surface reliably excited neurons in mouse cortical slices, demonstrating for the first time the feasibility of simultaneously activating multiple neurons or neuronal compartments with patterns mapped onto separate dendrites, at low excitation power densities $\left(0.3-0.6 \mathrm{~mW} / \mu \mathrm{m}^{2}\right)$. The decreased power density enabled better preservation of the lateral and axial resolution compared to scanning methods that direct the power used to a near diffraction-limited spot (Figure 4D). Moreover, the broad focal volume of large-surface spots, and the increased out-of-focus pulse duration by using TF reduces the chances to cause photodamage, even if we have to increase the total average power that is sent to the sample (Hopt and Neher, 2001) with cells sustaining to long-duration illumination protocols (Papagiakoumou et al., 2010).

\section{Perspectives and outlook}

Optogenetics has been introduced in the field of neuroscience during the last decade and it has been quickly adopted by scientists. Efforts to expand optogenetic tool capabilities have spurred innovations in genomic tool discovery, molecular engineering, opsin targeting and optical-device development. Although numerous optogenetic studies have provided important insights into neural function and dysfunction, complex interrogation and manipulation of neural circuits demands more flexible, controllable and spatiotemporally precise illumination methods, implementable in 2PE. Despite the progress in optical developments for optogenetics over the 
past five years, scientists continue to improve their performances by encountering the limitations of the techniques.

An important issue, for instance, when working in depth, both in vitro and in vivo, is scattering induced by the tissue. 2PE has become the most widely implemented method thus far to overcome scattering, as the scattered part of the beam, weak and temporally stretched outside the focus, contributes little to the 2PE signal. In addition, the longer wavelength used decreases scattering (Oheim et al., 2001). Even so, the signal decreases exponentially with depth $\left(S_{2 P E} \propto e^{-2 z / \ell_{s}}\right.$, where $\ell_{s}$ is the mean free path between two scattering events, or else scattering length; Helmchen and Denk, 2005). Moreover, scattering strongly distorts the optical wavefront of the excitation beam resulting in aberrated spots or, in the case of large excitation beams, interference of ballistic photons from adjacent illumination areas transform the intensity profile into a speckle pattern. The latter effect can also decrease the axial resolution , especially for temporally focused beams (Dana and Shoham, 2011, 2012a). Adaptive optics can improve propagation of diffraction-limited spots through scattering samples (Débarre et al., 2009; Katz et al., 2011; Rueckel et al., 2006; Vellekoop and Mosk, 2007), which has been especially useful for morphological imaging and other highly aberration sensitive applications. However, the limits on temporal resolution imposed by lengthy computation times have thus far outweighed potential benefits of utilizing adaptive optics for optogenetics applications.

Interestingly, recent experimental and theoretical studies on the propagation of temporally focused large Gaussian beams (Dana and Shoham, 2011, 2012a) and extended GPC-generated patterns through scattering media (Papagiakoumou et al., 2013) revealed that even fine details of the excitation shape are well preserved deep in scattering tissue, maintaining good axial confinement up to 1-2 scattering lengths. The robustness to scattering of temporally focused patterns can be attributed to the spectral "self-healing": each spectral component dispersed by the grating acquires its own speckle pattern, since it propagates through slightly different optical path than the rest of the spectral frequencies in the scattering medium. These uncorrelated speckle patterns interfere, smoothing out the intensity profile (Oron et al., 2012; Papagiakoumou et al., 2013). Thus, temporally focused patterns propose a powerful method to overcome scattering without introducing extra computation time or power losses.

An interesting application of patterned illumination can be for in vivo activation in freely moving animals with flexible fiber bundle image-transferring endoscopes (Bozinovic et al., 2008). Fiber transmission of intensity patterns provides a more practical solution than transmitting the phase map because multi-mode fibers randomize the phase, amplitude and polarization of the transmitted light. However, developers recently succeeded in transmitting CGHs through multimode optical fibers by implementing a direct search algorithm to modulate the phase, concentrating the output light power to one or more spots (Di Leonardo and Bianchi, 2011). 
CGH transmission with improved efficiency was also achieved with an optimization algorithm based on decomposition of the initial laser field at the SLM plane into a series of orthogonal modes each corresponding to a different square region on the SLM (Čižmár and Dholakia, 2011, 2012). Although interesting, the phase map transfer approaches are more complicated to implement than direct image pattern transfer and, in addition, highly sensitive to fiber movements, hindering adaption for in vivo applications.

Simultaneous 3D photoactivation and imaging of neuronal activity in separate planes poses another frontier at the nexus of wavefront shaping and optogenetics. Decoupling the imaging and activation planes would grant access to circuits and structures elaborated in three dimensions, such as dendritic arbors, enabling optical activation and monitoring of responses simultaneously in separate planes. This is not a trivial task, however, since typically both imaging and photoactivation are performed through the same objective lens. $\mathrm{DH}$, once more, can provide an elegant solution. Phase holograms can be modified to incorporate a 'lens effect', acting in practice as if we had placed an additional lens at the back focal plane of the objective, with which we can rapidly scan the z-position of an excitation pattern without physically modifying the optical components (Dal Maschio et al., 2011; Go et al., 2012; Packer et al., 2012; Yang et al., 2011; Zahid et al., 2010). Hence, the excitation patterned can be holographically scanned in $\mathrm{z}$ to precisely compensate the objective or stage scanning motion used for 3D imaging. Alternatively, DH can be combined with remote focusing (Botcherby et al., 2007), in which fluorescence imaging is performed by a second microscope objective symmetrical to the principal objective, recreating an aberration-free 3D image of the sample. In the combined system 3D holographic photoactivation utilizes the principal objective, while the plane imaged onto the camera is selected independently, with a mirror moving in the remote space (Anselmi et al., 2011). This system combining DH with remote focusing was used for $1 \mathrm{P}$ uncaging, but remote focusing alone has been implemented for $2 \mathrm{P}$ imaging by coupling an infrared laser beam to the remote unit, scanning laterally with a pair of galvanometer-driven mirrors and axially by displacing the remote mirror (Botcherby et al., 2012).

TF offers an alternative method to axially scan the laser beam remotely. Considering TF in the frequency domain, that is, based on the angular dispersion of the different spectral components, we can axially shift the TF plane by adding a quadratic spectral phase, analogous to the 'lens effect' for $\mathrm{DH}$, in which the focal plane is shifted via a quadratic spatial phase. A quadratic spectral phase involves introduction of a temporal delay, or 'chirp', between the frequencies comprising the spectral bandwidth of the femtosecond pulse before dispersing the spectral frequencies with the grating. Pre-chirping the frequencies results in recombination of the spectral components at a plane offset from the nominal objective focal plane (Durst et al., 2006). This phenomenon has been experimentally realized by chirping the beam with a pair of prisms (Durst et al., 2006, 2008), remotely with a pair of AODs (Du et al., 2009), a folded grating 
pair and a piezo-bimorph mirror (Straub et al., 2011) or by replacing the standard TF diffraction grating with a dual-prism grating (DPGrism) comprised of two prisms and a transmission diffraction grating (Dana and Shoham, 2012b). Lastly, we can achieve more flexible control of the laser pulse shape by modulating the spectral phase with a SLM at the Fourier plane of the TF telescope (Suchowski et al., 2006).

Finally, in parallel with the development of optogenetic tools, another class of genetically encoded molecular sensitizers has been presented that enables modulation of neuronal activity in response to temperature changes, so-called 'thermogenetic' tools (Bernstein et al., 2011). Thermogenetic approaches, similar to optogenetics, can be used for activation or for inhibition of neuronal activity. Since temperature affects all physiological processes, its use as a tool to control neuronal activity poses particular challenges, especially for applications in mammalian models (Bernstein et al., 2011), that are not going to be discussed here. Thermogenetics show advantages, like the possibility to stimulate simultaneously and non-invasively different regions of the brain at great depths, and limitations such as the lack of the millisecond temporal resolution achieved by optogenetics. Thermogenetics also lack of excitation spatial precision, since thermal stimulation, thus far, is done by ambient changes of temperature. This stimulation method has restricted thermogenetic application in Drosophila. However, development of methods that could extend their application to other animals (e.g. localized heating by using ultrasounds or lasers) could make them a complementary tool to optogenetic approaches in order to enhance the characterization and manipulation of mammalian circuit properties and functions.

In this review the illumination methods used so far in optogenetic applications were examined, discussing the advantages and limitations of each, in order to help readers to choose strategies well adapted to their specific experimental parameters. As evidenced throughout, the method selected from among those presented should be well adapted to the reporter and desired scale (sub-cellular, cellular, networks, in vivo).

\section{Acknowledgements}

I thank A.J. Foust, A. Bègue, E. Ronzitti and V. Emiliani for useful comments on the manuscript. I declare no conflict of interest.

\section{References}

${ }^{*}$ Articles of special interest

Acker, C.D., Yan, P., and Loew, L.M. (2011). Single-voxel recording of voltage transients in dendritic spines. Biophys. J. 101, L11-L13. 
Adamantidis, A.R., Zhang, F., Aravanis, A.M., Deisseroth, K., and De Lecea, L. (2007). Neural substrates of awakening probed with optogenetic control of hypocretin neurons. Nature 450, 420-424.

*Akemann, W., Mutoh, H., Perron, A., Rossier, J., and Knöpfel, T. (2010). Imaging brain electric signals with genetically targeted voltage-sensitive fluorescent proteins. Nat. Methods 7, 643-649.

Akerboom, J., Chen, T.-W., Wardill, T.J., Tian, L., Marvin, J.S., Mutlu, S., Calderón, N.C., Esposti, F., Borghuis, B.G., Sun, X.R., et al. (2012). Optimization of a GCaMP Calcium Indicator for Neural Activity Imaging. J. Neurosci. 32, 13819-13840.

*Andrasfalvy, B.K., Zemelman, B. V, Tang, J., and Vaziri, A. (2010). Two-photon single-cell optogenetic control of neuronal activity by sculpted light. Proc. Natl. Acad. Sci. U. S. A. 107, 11981-11986.

Anikeeva, P., Andalman, A.S., Witten, I., Warden, M., Goshen, I., Grosenick, L., Gunaydin, L. a, Frank, L.M., and Deisseroth, K. (2012). Optetrode: a multichannel readout for optogenetic control in freely moving mice. Nat. Neurosci. 15, 163-170.

*Anselmi, F., Ventalon, C., Bègue, A., Ogden, D., and Emiliani, V. (2011). Three-dimensional imaging and photostimulation by remote-focusing and holographic light patterning. Proc. Natl. Acad. Sci. U. S. A. 108, 19504-19509.

Aravanis, A.M., Wang, L.P., Zhang, F., Meltzer, L.A., Mogri, M.Z., Schneider, M.B., and Deisseroth, K. (2007). An optical neural interface: in vivo control of rodent motor cortex with integrated fiberoptic and optogenetic technology. J. Neural Eng. 4, S143-S156.

Arrenberg, A.B., Stainier, D.Y.R., Baier, H., and Huisken, J. (2010). Optogenetic control of cardiac function. Science 330, 971-974.

Arrizón, V., and Testorf, M. (1997). Efficiency limit of spatially quantized Fourier array illuminators. Opt. Lett. 22, 197-199.

Ashkin, A., and Dziedzic, J.M. (1987). Optical trapping and manipulation of viruses and bacteria. Science 235, 1517.

Ashkin, A., Dziedzic, J.M., Bjorkholm, J.E., and Chu, S. (1986). Observation of a single-beam gradient force optical trap for dielectric particles. Opt. Lett. 11, 288-290.

Axelrod, D. (1981). Cell-substrate contacts illuminated by total internal reflection fluorescence. J. Cell Biol. 89, 141-145.

Banghart, M., Borges, K., Isacoff, E., Trauner, D., and Kramer, R.H. (2004). Light-activated ion channels for remote control of neuronal firing. Nat. Neurosci. 7, 1381-1386.

Bernstein, J., Garrity, P., and Boyden, E. (2011). Optogenetics and thermogenetics: technologies for controlling the activity of targeted cells within intact neural circuits. Curr. Opin. Neurobiol. 22, 61-71. 
Botcherby, E.J., Juskaitis, R., Booth, M.J., and Wilson, T. (2007). Aberration-free optical refocusing in high numerical aperture microscopy. Opt. Lett. 32, 2007-2009.

Botcherby, E.J., Smith, C.W., Kohl, M.M., Débarre, D., Booth, M.J., Juskaitis, R., Paulsen, O., and Wilson, T. (2012). Aberration-free three-dimensional multiphoton imaging of neuronal activity at kHz rates. Proc.

Natl. Acad. Sci. U. S. A. 109, 2919-2924.

Bozinovic, N., Ventalon, C., Ford, T., and Mertz, J. (2008). Fluorescence endomicroscopy with structured illumination. Opt. Express 16, 4603-4610.

Bruegmann, T., Malan, D., Hesse, M., Beiert, T., Fuegemann, C.J., Fleischmann, B.K., and Sasse, P. (2010). Optogenetic control of heart muscle in vitro and in vivo. Nat. Methods 7, 897-900.

Bundschuh, S.T., Zhu, P., Schärer, Y.-P.Z., and Friedrich, R.W. (2012). Dopaminergic modulation of mitral cells and odor responses in the zebrafish olfactory bulb. J. Neurosci. 32, 6830-6840.

Busskamp, V., Duebel, J., Balya, D., Fradot, M., Viney, T.J., Siegert, S., Groner, A.C., Cabuy, E., Forster, V., Seeliger, M., et al. (2010). Genetic reactivation of cone photoreceptors restores visual responses in retinitis pigmentosa. Science 329, 413-417.

Buzsáki, G. (2004). Large-scale recording of neuronal ensembles. Nat. Neurosci. 7, 446-451.

Callaway, E.M., and Katz, L.C. (1993). Photostimulation using caged glutamate reveals functional circuitry in living brain slices. Proc. Natl. Acad. Sci. U. S. A. 90, 7661-7665.

Cardin, J. a, Carlén, M., Meletis, K., Knoblich, U., Zhang, F., Deisseroth, K., Tsai, L.-H., and Moore, C.I. (2009). Driving fast-spiking cells induces gamma rhythm and controls sensory responses. Nature 459, 663-667.

Chanda, B., Blunck, R., Faria, L.C., Schweizer, F.E., Mody, I., and Bezanilla, F. (2005). A hybrid approach to measuring electrical activity in genetically specified neurons. Nat. Neurosci. 8, 1619-1626.

Chen, Q., Cichon, J., Wang, W., Qiu, L., Lee, S.-J.R., Campbell, N.R., DeStefino, N., Goard, M.J., Fu, Z., Yasuda, R., et al. (2012). Imaging Neural Activity Using Thy1-GCaMP Transgenic Mice. Neuron 76, 297308.

Čižmár, T., and Dholakia, K. (2011). Shaping the light transmission through a multimode optical fibre: complex transformation analysis and applications in biophotonics. Opt. Express 19, 18871-18884.

Čižmár, T., and Dholakia, K. (2012). Exploiting multimode waveguides for pure fibre-based imaging. Nat. Commun. 3, 1027.

Collot, M., Loukou, C., Yakovlev, A. V, Wilms, C.D., Li, D., Evrard, A., Zamaleeva, A.I., Bourdieu, L., Leger, J.-F., Ropert, N., et al. (2012). Calcium Rubies: a family of red-emitting functionalizable indicators for twophoton Ca2+ imaging. J. Am. Chem. Soc. 134, 14923-14931. 
Curtis, J.E., Koss, B.A., and Grier, D.G. (2002). Dynamic holographic optical tweezers. Opt. Commun. 207, 169.

"Dal Maschio, M., Difato, F., Beltramo, R., Blau, A., Benfenati, F., and Fellin, T. (2010). Simultaneous twophoton imaging and photo-stimulation with structured light illumination. Opt. Express 18, 18720-18731.

Dal Maschio, M., De Stasi, A.M., Benfenati, F., and Fellin, T. (2011). Three-dimensional in vivo scanning microscopy with inertia-free focus control. Opt. Lett. 36, 3503-3505.

*Dana, H., and Shoham, S. (2011). Numerical evaluation of temporal focusing characteristics in transparent and scattering media. Opt. Express 19, 4937-4948.

Dana, H., and Shoham, S. (2012a). Numerical evaluation of temporal focusing characteristics in transparent and scattering media: erratum. Opt. Express 20, 28281.

Dana, H., and Shoham, S. (2012b). Remotely scanned multiphoton temporal focusing by axial grism scanning. Opt. Lett. 37, 2913-2915.

'Daria, V.R., Stricker, C., Bowman, R., Redman, S., and Bachor, H.A. (2009). Arbitrary multisite twophoton excitation in four dimensions. Appl. Phys. Lett. 95, 93701.

Débarre, D., Botcherby, E., and Watanabe, T. (2009). Image-based adaptive optics for two-photon microscopy. Opt. Lett. 34, 2495-2497.

Degenaar, P., Grossman, N., Memon, M.A., Burrone, J., Dawson, M., Drakakis, E., Neil, M., and Nikolic, K. (2009). Optobionic vision--a new genetically enhanced light on retinal prosthesis. J. Neural Eng. 6, 035007.

Deisseroth, K., Feng, G., Majewska, A.K., Miesenböck, G., Ting, A., and Schnitzer, M.J. (2006). Nextgeneration optical technologies for illuminating genetically targeted brain circuits. J. Neurosci. 26, 10380-10386.

Denk, W., Strickler, J.H., and Webb, W.W. (1990). Two-photon laser scanning fluorescence microscopy. Science 248, 73-76.

Depuy, S.D., Kanbar, R., Coates, M.B., Stornetta, R.L., and Guyenet, P.G. (2011). Control of breathing by raphe obscurus serotonergic neurons in mice. J. Neurosci. 31, 1981-1990.

Dhawale, A.K., Hagiwara, A., Bhalla, U.S., Murthy, V.N., and Albeanu, D.F. (2010). Non-redundant odor coding by sister mitral cells revealed by light addressable glomeruli in the mouse. Nat. Neurosci. 13, 1404-1412.

Dimitrov, D., He, Y., Mutoh, H., Baker, B.J., Cohen, L., Akemann, W., and Knöpfel, T. (2007). Engineering and characterization of an enhanced fluorescent protein voltage sensor. PLoS One 2, e440.

Dombeck, D. a, Harvey, C.D., Tian, L., Looger, L.L., and Tank, D.W. (2010). Functional imaging of hippocampal place cells at cellular resolution during virtual navigation. Nat. Neurosci. 13, 1433-1440. 
Du, R., Bi, K., Zeng, S., Li, D., Xue, S., and Luo, Q. (2009). Analysis of fast axial scanning scheme using temporal focusing with acousto-optic deflectors. J. Mod. Opt. 56, 81-84.

Durst, M.E., Zhu, G., and Xu, C. (2006). Simultaneous spatial and temporal focusing for axial scanning. Opt. Express 14, 12243-12254.

Durst, M.E., Zhu, G., and Xu, C. (2008). Simultaneous spatial and temporal focusing in nonlinear microscopy. Opt. Commun. 281, 1796-1805.

Fan, G.Y., Fujisaki, H., Miyawaki, A., Tsay, R.K., Tsien, R.Y., and Ellisman, M.H. (1999). Video-rate scanning two-photon excitation fluorescence microscopy and ratio imaging with cameleons. Biophys. J. 76, 24122420.

Farber, I.C., and Grinvald, A. (1983). Identification of presynaptic neurons by laser photostimulation. Science 222, 1025-1027.

Feldbauer, K., Zimmermann, D., Pintschovius, V., Spitz, J., Bamann, C., and Bamberg, E. (2009). Channelrhodopsin-2 is a leaky proton pump. Proc. Natl. Acad. Sci. U. S. A. 106, 12317-12322.

Fisher, J. a N., Barchi, J.R., Welle, C.G., Kim, G.-H., Kosterin, P., Obaid, A.L., Yodh, A.G., Contreras, D., and Salzberg, B.M. (2008). Two-photon excitation of potentiometric probes enables optical recording of action potentials from mammalian nerve terminals in situ. J. Neurophysiol. 99, 1545-1553.

Fork, R. (1971). Laser stimulation of nerve cells in aplysia. Science 3, 907-908.

Fortin, D., Banghart, M., and Dunn, T. (2008). Photochemical control of endogenous ion channels and cellular excitability. Nat. Methods 5, 331-338.

Gasparini, S., and Magee, J.C. (2006). State-dependent dendritic computation in hippocampal CA1 pyramidal neurons. J. Neurosci. 26, 2088-2100.

Gerchberg, R.W., and Saxton, W.O. (1972). A pratical algorithm for the determination of the phase from image and diffraction pictures. Optik 35, 237-246.

Glückstad, J. (1996). Phase contrast image synthesis. Opt. Commun. 130, 225-230.

Glückstad, J., and Mogensen, P.C. (2001). Optimal phase contrast in common-path interferometry. Appl. Optics 40, 268-282.

Go, M.A., Ng, P.-F., Bachor, H. a, and Daria, V.R. (2011). Optimal complex field holographic projection. Opt. Lett. 36, 3073-3075.

Go, M.A., Stricker, C., Redman, S., Bachor, H.-A., and Daria, V.R. (2012). Simultaneous multi-site twophoton photostimulation in three dimensions. J. Biophotonics 5, 745-753.

Göbel, W., Kampa, B.M., and Helmchen, F. (2006). Imaging cellular network dynamics in three dimensions using fast 3D laser scanning. Nat. Methods 4, 73-79. 
*Golan, L., and Shoham, S. (2009). Speckle elimination using shift-averaging in high-rate holographic projection. Opt. Express 17, 1330-1339.

Golan, L., Reutsky, I., Farah, N., and Shoham, S. (2009). Design and characteristics of holographic neural photo-stimulation systems. J. Neural Eng. 6, 66004.

*Gradinaru, V., Thompson, K.R., Zhang, F., Mogri, M., Kay, K., Schneider, M.B., and Deisseroth, K. (2007). Targeting and readout strategies for fast optical neural control in vitro and in vivo. J. Neurosci. 27, 14231-14238.

*Grossman, N., Poher, V., Grubb, M.S., Kennedy, G.T., Nikolic, K., McGovern, B., Palmini, R.B., Gong, Z., Drakakis, E.M., Neil, M.A., et al. (2010). Multi-site optical excitation using ChR2 and micro-LED array. J. Neural Eng. 7, 16004.

Guo, Z. V, Hart, A.C., and Ramanathan, S. (2009). Optical interrogation of neural circuits in Caenorhabditis elegans. Nat. Methods 6, 891-896.

Gutierrez, D. V, Mark, M.D., Masseck, O., Maejima, T., Kuckelsberg, D., Hyde, R. a, Krause, M., Kruse, W., and Herlitze, S. (2011). Optogenetic control of motor coordination by Gi/o protein-coupled vertebrate rhodopsin in cerebellar Purkinje cells. J. Biol. Chem. 286, 25848-25858.

Hamill, O.P., Marty, A., Neher, E., Sakmann, B., and Sigworth, F.J. (1981). Improved patch-clamp techniques for high-resolution current recording from cells and cell-free membrane patches. Pflügers Arch. 391, 85-100.

Heim, R., Prasher, D.C., and Tsien, R.Y. (1994). Wavelength mutations and posttranslational autoxidation of green fluorescent protein. Proc. Natl. Acad. Sci. U. S. A. 91, 12501-12504.

Heim, R., Cubitt, A.B., and Tsien, R.Y. (1995). Improved green fluorescence. Nature 373, 663-664.

${ }^{*}$ Helmchen, F., and Denk, W. (2005). Deep tissue two-photon microscopy. Nat. Methods 2, 932-940.

Hirase, H., Nikolenko, V., Goldberg, J.H., and Yuste, R. (2002). Multiphoton stimulation of neurons. J. Neurobiol. 51, 237-247.

Hopt, a, and Neher, E. (2001). Highly nonlinear photodamage in two-photon fluorescence microscopy.

Biophys. J. 80, 2029-2036.

Huber, D., Petreanu, L., Ghitani, N., Ranade, S., Hromadka, T., Mainen, Z., and Svoboda, K. (2008). Sparse optical microstimulation in barrel cortex drives learned behaviour in freely moving mice. Nature 451, 6164.

Jesacher, A., and Booth, M.J. (2010). Parallel direct laser writing in three dimensions with spatially dependent aberration correction. Opt. Express 18, 21090-21099.

Jesacher, A., Maurer, C., Schwaighofer, A., Bernet, S., and Ritsch-Marte, M. (2008). Full phase and amplitude control of holographic optical tweezers with high efficiency. Opt. Express 16, 4479-4486. 
Kasparov, S., and Herlitze, S. (2013). Optogenetics at a crossroads? Exp. Physiol. 00, 1-2.

Katona, G., Szalay, G., Maák, P., Kaszás, A., Veress, M., Hillier, D., Chiovini, B., Vizi, E.S., Roska, B., and Rózsa, B. (2012). Fast two-photon in vivo imaging with three-dimensional random-access scanning in large tissue volumes. Nat. Methods 9, 201-208.

${ }^{*}$ Katz, L.C., and Dalva, M.B. (1994). Scanning laser photostimulation: a new approach for analyzing brain circuits. J. Neurosci. Meth. 54, 205-218.

"Katz, O., Small, E., Bromberg, Y., and Silberberg, Y. (2011). Focusing and compression of ultrashort pulses through scattering media. Nature Photon. 5, 372-377.

Kleinlogel, S., Feldbauer, K., Dempski, R.E., Fotis, H., Wood, P.G., Bamann, C., and Bamberg, E. (2011). Ultra light-sensitive and fast neuronal activation with the $\mathrm{Ca}^{2}+$-permeable channelrhodopsin CatCh. Nat. Neurosci. 14, 513-518.

Knapczyk, M., and Krishnan, A. (2005). High-resolution pulse shaper based on arrays of digital micromirrors. IEEE Photonic. Tech. L. 17, 2200-2202.

Knöpfel, T. (2012). Genetically encoded optical indicators for the analysis of neuronal circuits. Nat. Rev. Neurosci. 13, 687-700.

Ko, H., Hofer, S.B., Pichler, B., Buchanan, K. a, Sjöström, P.J., and Mrsic-Flogel, T.D. (2011). Functional specificity of local synaptic connections in neocortical networks. Nature 473, 87-91.

Kravitz, A. V, Freeze, B.S., Parker, P.R., Kay, K., Thwin, M.T., Deisseroth, K., and Kreitzer, A.C. (2010). Regulation of parkinsonian motor behaviours by optogenetic control of basal ganglia circuitry. Nature 466, 622-626.

Kuhn, B., Denk, W., and Bruno, R.M. (2008). In vivo two-photon voltage-sensitive dye imaging reveals top-down control of cortical layers 1 and 2 during wakefulness. Proc. Natl. Acad. Sci. U. S. A. 105, 75887593.

Lee, J.H., Durand, R., Gradinaru, V., Zhang, F., Goshen, I., Kim, D.-S., Fenno, L.E., Ramakrishnan, C., and Deisseroth, K. (2010). Global and local fMRI signals driven by neurons defined optogenetically by type and wiring. Nature 465, 788-792.

*Leifer, A.M., Fang-Yen, C., Gershow, M., Alkema, M.J., and Samuel, A.D.T. (2011). Optogenetic manipulation of neural activity in freely moving Caenorhabditis elegans. Nat. Methods 8, 147-152.

Di Leonardo, R., and Bianchi, S. (2011). Hologram transmission through multi-mode optical fibers. Opt. Express 19, 1867-1869.

Di Leonardo, R., lanni, F., and Ruocco, G. (2007). Computer generation of optimal holograms for optical trap arrays. Opt. Express 15, 1913-1922. 
*Levitz, J., Pantoja, C., Gaub, B., Janovjak, H., Reiner, A., Hoagland, A., Schoppik, D., Kane, B., Stawski, P., Schier, A.F., et al. (2013). Optical control of metabotropic glutamate receptors. Nat. Neurosci.

Li, D., Hérault, K., Isacoff, E.Y., Oheim, M., and Ropert, N. (2012). Optogenetic activation of LiGluRexpressing astrocytes evokes anion channel-mediated glutamate release. J. Physiol. 590, 855-873.

Li, N., Downey, J.E., Bar-Shir, A., Gilad, A. a, Walczak, P., Kim, H., Joel, S.E., Pekar, J.J., Thakor, N. V, and Pelled, G. (2011). Optogenetic-guided cortical plasticity after nerve injury. Proc. Natl. Acad. Sci. U. S. A. $108,8838-8843$.

Liesener, J., Reicherter, M., Haist, T., and Tiziani, H.J. (2000). Multi-functional optical tweezers using computer-generated holograms. Opt. Commun. 185, 77-82.

Lin, J.Y., Lin, M.Z., Steinbach, P., and Tsien, R.Y. (2009). Characterization of engineered channelrhodopsin variants with improved properties and kinetics. Biophys. J. 96, 1803-1814.

Liu, X., Ramirez, S., Pang, P.T., Puryear, C.B., Govindarajan, A., Deisseroth, K., and Tonegawa, S. (2012). Optogenetic stimulation of a hippocampal engram activates fear memory recall. Nature 484, 381-385.

Losavio, B.E., Iyer, V., and Saggau, P. (2009). Two-photon microscope for multisite microphotolysis of caged neurotransmitters in acute brain slices. J. Biomed. Opt. 14, 064033.

Losonczy, A., Zemelman, B. V, Vaziri, A., and Magee, J.C. (2010). Network mechanisms of theta related neuronal activity in hippocampal CA1 pyramidal neurons. Nat. Neurosci. 13, 967-972.

"Lutz, C., Otis, T.S., DeSars, V., Charpak, S., Digregorio, D.A., and Emiliani, V. (2008). Holographic photolysis of caged neurotransmitters. Nat. Methods 5, 821-827.

Mao, T., O'Connor, D.H., Scheuss, V., Nakai, J., and Svoboda, K. (2008). Characterization and subcellular targeting of GCaMP-type genetically-encoded calcium indicators. PLoS One 3, e1796.

Matar, S., Golan, L., and Shoham, S. (2011). Reduction of two-photon holographic speckle using shiftaveraging. Opt. Express 19, 25891-25899.

Mattis, J., Tye, K.M., Ferenczi, E. a, Ramakrishnan, C., Shea, D.J.O., Prakash, R., Gunaydin, L. a, Hyun, M., Fenno, L.E., Gradinaru, V., et al. (2012). Principles for applying optogenetic tools derived from direct comparative analysis of microbial opsins. Nat. Methods 9, 159-172.

McAlinden, N., Massoubre, D., Richardson, E., Gu, E., Sakata, S., Dawson, M.D., and Mathieson, K. (2013). Thermal and optical characterization of micro-LED probes for in vivo optogenetic neural stimulation. Opt. Lett. 38, 992-994.

"Miesenböck, G. (2009). The optogenetic catechism. Science 326, 395-399.

Mohanty, S.K., Reinscheid, R.K., Liu, X., Okamura, N., Krasieva, T.B., and Berns, M.W. (2008). In-Depth Activation of Channelrhodopsin 2-Sensitized Excitable Cells with High Spatial Resolution Using TwoPhoton Excitation with a Near-Infrared Laser Microbeam Cell culture. Biophys. J. 95, 3916-3926. 
Nagel, G., Szellas, T., Huhn, W., Kateriya, S., Adeishvili, N., Berthold, P., Ollig, D., Hegemann, P., and Bamberg, E. (2003). Channelrhodopsin-2, a directly light-gated cation-selective membrane channel. Proc. Natl. Acad. Sci. U. S. A. 100, 13940-13945.

Nagel, G., Brauner, M., Liewald, J.F., Adeishvili, N., Bamberg, E., and Gottschalk, A. (2005). Light activation of channelrhodopsin- 2 in excitable cells of Caenorhabditis elegans triggers rapid behavioral responses. Curr. Biol. 15, 2279-2284.

Nakai, J., Ohkura, M., and Imoto, K. (2001). A high signal-to-noise $\mathrm{Ca}(2+)$ probe composed of a single green fluorescent protein. Nat. Biotechnol. 19, 137-141.

Nguyen, Q.T., Callamaras, N., Hsieh, C., and Parker, I. (2001). Construction of a two-photon microscope for video-rate $\mathrm{Ca}(2+)$ imaging. Cell Calcium 30, 383-393.

"Nikolenko, V., Watson, B.O., Araya, R., Woodruff, A., Peterka, D.S., and Yuste, R. (2008). SLM Microscopy: Scanless Two-Photon Imaging and Photostimulation with Spatial Light Modulators. Front. Neural Circuit. 2, 5.

Oheim, M., Beaurepaire, E., Chaigneau, E., Mertz, J., and Charpak, S. (2001). Two-photon microscopy in brain tissue: parameters influencing the imaging depth. J. Neurosci. Meth. 111, 29-37.

Oron, D., and Silberberg, Y. (2005). Harmonic generation with temporally focused ultrashort pulses. J. Opt. Soc. Am. B 22, 2660-2663.

*Oron, D., Tal, E., and Silberberg, Y. (2005). Scanningless depth-resolved microscopy. Opt. Express 13, 1468-1476.

*Oron, D., Papagiakoumou, E., Anselmi, F., and Emiliani, V. (2012). Two-photon optogenetics. Prog. Brain Res. 196, 119-143.

Otsu, Y., Bormuth, V., Wong, J., Mathieu, B., Dugue, G.P., Feltz, A., and Dieudonne, S. (2008). Optical monitoring of neuronal activity at high frame rate with a digital random-access multiphoton (RAMP) microscope. J. Neurosci. Meth. 173, 259-270.

*Packer, A.M., Peterka, D.S., Hirtz, J.J., Prakash, R., Deisseroth, K., and Yuste, R. (2012). Two-photon optogenetics of dendritic spines and neural circuits. Nat. Methods 9, 1202-1205.

Palima, D., and Daria, V.R. (2006). Effect of spurious diffraction orders in arbitrary multifoci patterns produced via phase-only holograms. Appl. Optics 45, 6689-6693.

Palima, D., and Daria, V.R. (2007). Holographic projection of arbitrary light patterns with a suppressed zero-order beam. Appl. Optics 46, 4197-4201.

Palima, D., and Glückstad, J. (2008). Comparison of generalized phase contrast and computer generated holography for laser image projection. Opt. Express 16, 5338-5349. 
Panda, S., Nayak, S.K., Campo, B., Walker, J.R., Hogenesch, J.B., and Jegla, T. (2005). Illumination of the melanopsin signaling pathway. Science 307, 600-604.

*Papagiakoumou, E., de Sars, V., Oron, D., and Emiliani, V. (2008). Patterned two-photon illumination by spatiotemporal shaping of ultrashort pulses. Opt. Express 16, 22039-22047.

Papagiakoumou, E., de Sars, V., Emiliani, V., and Oron, D. (2009). Temporal focusing with spatially modulated excitation. Opt. Express 17, 5391-5401.

"Papagiakoumou, E., Anselmi, F., Bègue, A., de Sars, V., Glückstad, J., Isacoff, E.Y., and Emiliani, V. (2010). Scanless two-photon excitation of channelrhodopsin-2. Nat. Methods 7, 848-854.

*Papagiakoumou, E., Bègue, A., Leshem, B., Schwartz, O., Stell, B.M., Bradley, J., Oron, D., and Emiliani, V. (2013). Functional patterned multiphoton excitation deep inside scattering tissue. Nature Photon. 7, 274-278.

Petreanu, L., Huber, D., Sobczyk, A., and Svoboda, K. (2007). Channelrhodopsin-2-assisted circuit mapping of long-range callosal projections. Nat. Neurosci. 10, 663-668.

Petreanu, L., Mao, T., Sternson, S.M., and Svoboda, K. (2009). The subcellular organization of neocortical excitatory connections. Nature 457, 1142-1145.

Piccolino, M. (2006). Luigi Galvani's path to animal electricity. C. R. Biol. 329, 303-318.

Polosukhina, A., Litt, J., Tochitsky, I., Nemargut, J., Sychev, Y., De Kouchkovsky, I., Huang, T., Borges, K., Trauner, D., Van Gelder, R.N., et al. (2012). Photochemical Restoration of Visual Responses in Blind Mice. Neuron 75, 271-282.

"Prakash, R., Yizhar, O., Grewe, B., Ramakrishnan, C., Wang, N., Goshen, I., Packer, A.M., Peterka, D.S., Yuste, R., Schnitzer, M.J., et al. (2012). Two-photon optogenetic toolbox for fast inhibition, excitation and bistable modulation. Nat. Methods 9, 1171-1179.

Qiu, X., Kumbalasiri, T., Carlson, S.M., Wong, K.Y., Krishna, V., Provencio, I., and Berson, D.M. (2005). Induction of photosensitivity by heterologous expression of melanopsin. Nature 433, 745-749.

Reddy, G.D., and Saggau, P. (2005). Fast three-dimensional laser scanning scheme using acousto-optic deflectors. J. Biomed. Opt. 10, 64038.

Reddy, G.D., Kelleher, K., Fink, R., and Saggau, P. (2008). Three-dimensional random access multiphoton microscopy for functional imaging of neuronal activity. Nat. Neurosci. 11, 713-720.

Reicherter, M., Haist, T., Wagemann, E.U., and Tiziani, H.J. (1999). Optical particle trapping with computer-generated holograms written on a liquid-crystal display. Opt. Lett. 24, 608-610.

"Reutsky-Gefen, I., Golan, L., Farah, N., Schejter, A., Tsur, L., Brosh, I., and Shoham, S. (2013). Holographic optogenetic stimulation of patterned neuronal activity for vision restoration. Nat. Commun. 4, 1509. 
*Rickgauer, J.P., and Tank, D.W. (2009). Two-photon excitation of channelrhodopsin-2 at saturation. Proc. Natl. Acad. Sci. U. S. A. 106, 15025-15030.

Rochefort, N.L., Garaschuk, O., Milos, R.-I., Narushima, M., Marandi, N., Pichler, B., Kovalchuk, Y., and Konnerth, A. (2009). Sparsification of neuronal activity in the visual cortex at eye-opening. Proc. Natl. Acad. Sci. U. S. A. 106, 15049-15054.

Ronzitti, E., Guillon, M., De Sars, V., and Emiliani, V. (2012). LCoS nematic SLM characterization and modeling for diffraction efficiency optimization, zero and ghost orders suppression. Opt. Express 20, 17843-17855.

Royer, S., Zemelman, B. V, Barbic, M., Losonczy, A., Buzsáki, G., and Magee, J.C. (2010). Multi-array silicon probes with integrated optical fibers: light-assisted perturbation and recording of local neural circuits in the behaving animal. Eur. J. Neursci. 31, 2279-2291.

Rueckel, M., Mack-Bucher, J. a, and Denk, W. (2006). Adaptive wavefront correction in two-photon microscopy using coherence-gated wavefront sensing. Proc. Natl. Acad. Sci. U. S. A. 103, 17137-17142.

Sakai, S., Ueno, K., Ishizuka, T., and Yawo, H. (2013). Parallel and patterned optogenetic manipulation of neurons in the brain slice using a DMD-based projector. Neurosci. Res. 75, 59-64.

Salomé, R., Kremer, Y., Dieudonné, S., Léger, J.-F., Krichevsky, O., Wyart, C., Chatenay, D., and Bourdieu, L. (2006). Ultrafast random-access scanning in two-photon microscopy using acousto-optic deflectors. J. Neurosci. Meth. 154, 161-174.

Santos, M.D., Mohammadi, M.H., Yang, S., Liang, C.W., Kao, J.P.Y., Alger, B.E., Thompson, S.M., and Tang, C.-M. (2012). Dendritic hold and read: a gated mechanism for short term information storage and retrieval. PLoS One 7, e37542.

Scanziani, M., and Hausser, M. (2009). Electrophysiology in the age of light. Nature 461, 930-939.

Shoham, S., O'Connor, D.H., Sarkisov, D. V, and Wang, S.S. (2005). Rapid neurotransmitter uncaging in spatially defined patterns. Nat. Methods 2, 837-843.

Siegel, M., and Isacoff, E. (1997). A genetically encoded optical probe of membrane voltage. Neuron 19, 735-741.

Sohal, V.S., Zhang, F., Yizhar, O., and Deisseroth, K. (2009). Parvalbumin neurons and gamma rhythms enhance cortical circuit performance. Nature 459, 698-702.

Stirman, J.N., Crane, M.M., Husson, S.J., Wabnig, S., Schultheis, C., Gottschalk, A., and Lu, H. (2011). Realtime multimodal optical control of neurons and muscles in freely behaving Caenorhabditis elegans. Nat. Methods 8, 153-158.

Stirman, J.N., Crane, M.M., Husson, S.J., Gottschalk, A., and Lu, H. (2012). A multispectral optical illumination system with precise spatiotemporal control for the manipulation of optogenetic reagents. Nat. Protoc. 7, 207-220. 
Straub, A., Durst, M.E., and Xu, C. (2011). High speed multiphoton axial scanning through an optical fiber in a remotely scanned temporal focusing setup. Biomed. Opt. Express 2, 80-88.

Stuart, G.J., and Sakmann, B. (1994). Active propagation of somatic action potentials into neocortical pyramidal cell dendrites. Nature 367, 69-72.

Stuart, G., Schiller, J., and Sakmann, B. (1997). Action potential initiation and propagation in rat neocortical pyramidal neurons. J. Physiol. 505 ( Pt 3, 617-632.

Suchowski, H., Oron, D., Silberberg, Y., and Suchowski Oron, D., Silberberg, Y., H. (2006). Generation of a dark nonlinear focus by spatio-temporal coherent control. Opt. Commun. 264, 482-487.

Tian, L., Hires, S.A., Mao, T., Huber, D., Chiappe, M.E., Chalasani, S.H., Petreanu, L., Akerboom, J., McKinney, S. a, Schreiter, E.R., et al. (2009). Imaging neural activity in worms, flies and mice with improved GCaMP calcium indicators. Nat. Methods 6, 875-881.

Tsai, H.C., Zhang, F., Adamantidis, A., Stuber, G.D., Bonci, A., De Lecea, L., and Deisseroth, K. (2009). Phasic firing in dopaminergic neurons is sufficient for behavioral conditioning. Science 324, 1080-1084.

Vaziri, A., and Emiliani, V. (2012). Reshaping the optical dimension in optogenetics. Curr. Opin. Neurobiol. 22, 128-137.

Vellekoop, I.M., and Mosk, A.P. (2007). Focusing coherent light through opaque strongly scattering media. Opt. Lett. 32, 2309-2311.

Volgraf, M., Gorostiza, P., Numano, R., Kramer, R.H., Isacoff, E.Y., and Trauner, D. (2006). Allosteric control of an ionotropic glutamate receptor with an optical switch. Nat. Chem. Biol. 2, 47-52.

Wang, H., Peca, J., Matsuzaki, M., Matsuzaki, K., Noguchi, J., Qiu, L., Wang, D., Zhang, F., Boyden, E., Deisseroth, K., et al. (2007a). High-speed mapping of synaptic connectivity using photostimulation in Channelrhodopsin-2 transgenic mice. Proc. Natl. Acad. Sci. U. S. A. 104, 8143-8148.

Wang, J., Wagner, F., Borton, D. a, Zhang, J., Ozden, I., Burwell, R.D., Nurmikko, A. V, Van Wagenen, R., Diester, I., and Deisseroth, K. (2012). Integrated device for combined optical neuromodulation and electrical recording for chronic in vivo applications. J. Neural Eng. 9, 016001.

"Wang, K., Liu, Y., Li, Y., Guo, Y., Song, P., Zhang, X., Zeng, S., and Wang, Z. (2011). Precise spatiotemporal control of optogenetic activation using an acousto-optic device. PLoS One 6, e28468.

Wang, S., Szobota, S., Wang, Y., Volgraf, M., Liu, Z., Sun, C., Trauner, D., Isacoff, E.Y., and Zhang, X. (2007b). All optical interface for parallel, remote, and spatiotemporal control of neuronal activity. Nano Lett. 7, 3859-3863.

Watt, A.J., Cuntz, H., Mori, M., Nusser, Z., Sjöström, P.J., and Häusser, M. (2009). Traveling waves in developing cerebellar cortex mediated by asymmetrical Purkinje cell connectivity. Nat. Neurosci. 12, 463-473. 
Wilson, N.R., Runyan, C. a, Wang, F.L., and Sur, M. (2012). Division and subtraction by distinct cortical inhibitory networks in vivo. Nature 488, 343-348.

Wilt, B. a, Burns, L.D., Wei Ho, E.T., Ghosh, K.K., Mukamel, E. a, and Schnitzer, M.J. (2009). Advances in light microscopy for neuroscience. Annu. Rev. Neurosci. 32, 435-506.

Witten, I.B., Lin, S.-C., Brodsky, M., Prakash, R., Diester, I., Anikeeva, P., Gradinaru, V., Ramakrishnan, C., and Deisseroth, K. (2010). Cholinergic interneurons control local circuit activity and cocaine conditioning. Science 330, 1677-1681.

Wyart, C., Del Bene, F., Warp, E., Scott, E.K., Trauner, D., Baier, H., and Isacoff, E.Y. (2009). Optogenetic dissection of a behavioural module in the vertebrate spinal cord. Nature 461, 407-410.

Yan, P., Acker, C.D., Zhou, W.-L., Lee, P., Bollensdorff, C., Negrean, A., Lotti, J., Sacconi, L., Antic, S.D., Kohl, P., et al. (2012). Palette of fluorinated voltage-sensitive hemicyanine dyes. Proc. Natl. Acad. Sci. U. S. A. 109, 20443-20448.

*Yang, S., Papagiakoumou, E., Guillon, M., De Sars, V., Tang, C.M., and Emiliani, V. (2011). Threedimensional holographic photostimulation of the dendritic arbor. J. Neural Eng. 8, 46002.

Yizhar, O., Fenno, L.E., Davidson, T.J., Mogri, M., and Deisseroth, K. (2011). Optogenetics in neural systems. Neuron 71, 9-34.

Zahid, M., Velez-Fort, M., Papagiakoumou, E., Ventalon, C., Angulo, M.C., and Emiliani, V. (2010). Holographic photolysis for multiple cell stimulation in mouse hippocampal slices. PLoS One 5, e9431.

Zemelman, B., Lee, G., Ng, M., and Miesenböck, G. (2002). Selective photostimulation of genetically chARGed neurons. Neuron 33, 15-22.

"Zemelman, B. V, Nesnas, N., Lee, G. a, and Miesenbock, G. (2003). Photochemical gating of heterologous ion channels: remote control over genetically designated populations of neurons. Proc. Natl. Acad. Sci. U. S. A. 100, 1352-1357.

Zernike, F. (1955). How I discovered phase contrast. Science 121, 345-349.

Zhang, Y.P., and Oertner, T.G. (2007). Optical induction of synaptic plasticity using a light-sensitive channel. Nat. Methods 4, 139-141.

Zhang, F., Wang, L.P., Boyden, E.S., and Deisseroth, K. (2006). Channelrhodopsin-2 and optical control of excitable cells. Nat. Methods 3, 785-792.

Zhang, F., Wang, L.P., Brauner, M., Liewald, J.F., Kay, K., Watzke, N., Wood, P.G., Bamberg, E., Nagel, G., Gottschalk, A., et al. (2007). Multimodal fast optical interrogation of neural circuitry. Nature 446, 633639. 
"Zhang, F., Gradinaru, V., Adamantidis, A.R., Durand, R., Airan, R.D., De Lecea, L., and Deisseroth, K. (2010). Optogenetic interrogation of neural circuits: technology for probing mammalian brain structures. Nat. Protoc. 5, 439-456.

Zhang, J., Laiwalla, F., and Kim, J. (2009). Integrated device for optical stimulation and spatiotemporal electrical recording of neural activity in light-sensitized brain tissue. J. Neural Eng. 6, 055007.

Zhang, Y., Holbro, N., and Oertner, T.G. (2008). Optical induction of plasticity at single synapses reveals input-specific accumulation of alphaCaMKII. Proc. Natl. Acad. Sci. U. S. A. 105, 12039-12044.

*Zhu, G., Van Howe, J., Durst, M., Zipfel, W., and Xu, C. (2005). Simultaneous spatial and temporal focusing of femtosecond pulses. Opt. Express 13, 2153-2159.

Zhu, P., Fajardo, O., Shum, J., Zhang Schärer, Y.-P., and Friedrich, R.W. (2012). High-resolution optical control of spatiotemporal neuronal activity patterns in zebrafish using a digital micromirror device. Nat. Protoc. 7, 1410-1425.

Zipfel, W.R., Williams, R.M., and Webb, W.W. (2003). Nonlinear magic: multiphoton microscopy in the biosciences. Nat. Biotechnol. 21, 1369-1377.

Zorzos, A., Boyden, E.S., and Fonstad, C.G. (2010). A multi-waveguide implantable probe for light delivery to sets of distributed brain targets. Opt. Lett. 35, 4133-4135. 


\section{Figures}
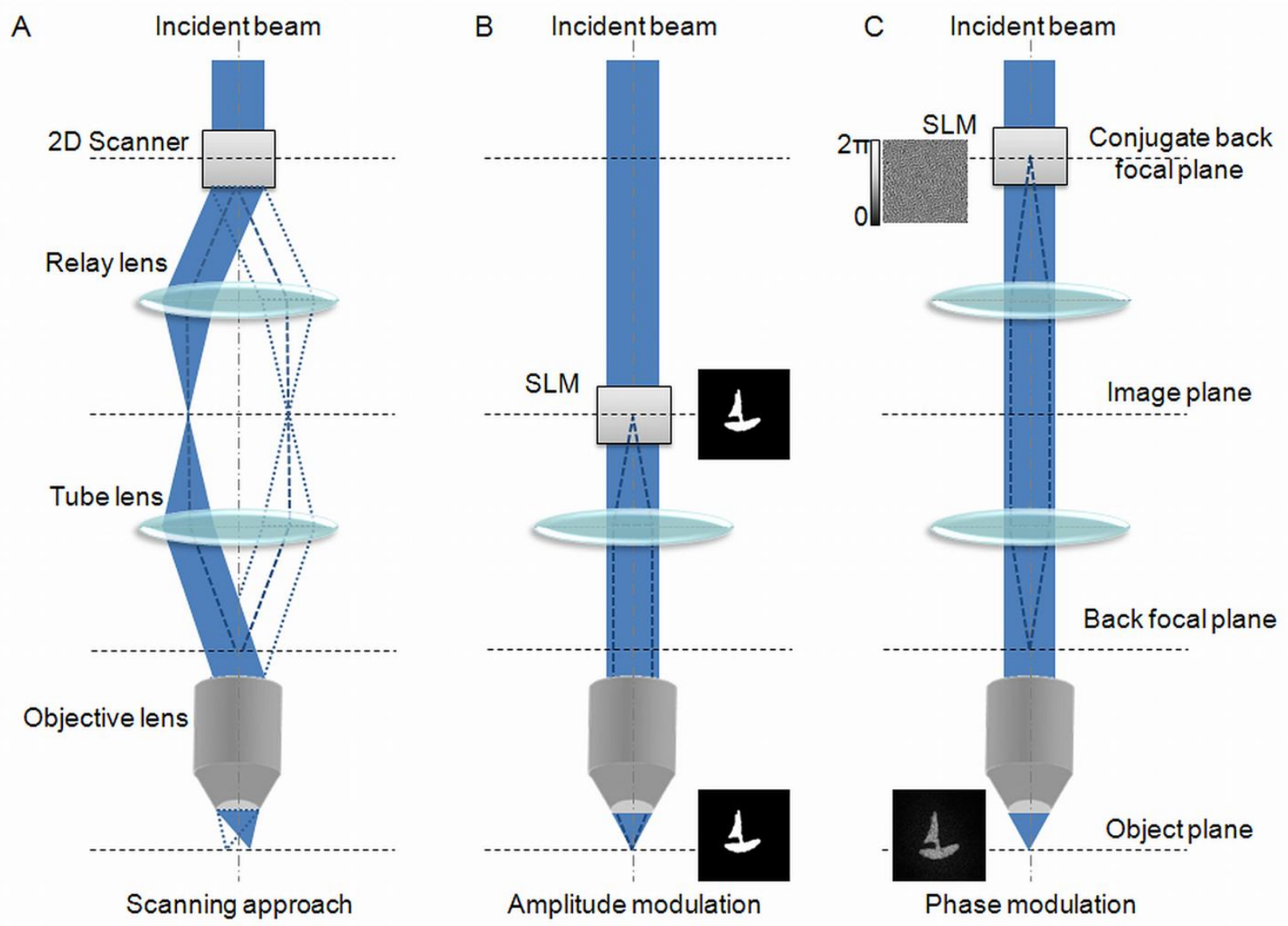

\section{Figure 1 | Laser illumination techniques}

(A) Schematic illustrating the principle of scanning approaches. The scanner (galvanometerdriven mirrors or AODs) is imaged onto the back focal plane of the microscope objective (blue dashed lines). In this manner angular deflections induced by the scanner to the beam, correspond to lateral translations in the focal plane of the objective. Light blue dotted lines show a different deflection angle of the beam. (B) Schematic of a typical setup for amplitude modulation. Each pixel of the SLM (e.g. a DMD) is imaged at the sample plane (blue dashed lines). The desired intensity pattern is addressed to the SLM and reproduced at the focal plane of the objective. (C) Schematic of a phase modulation setup. Each pixel of the SLM is imaged at the back focal plane of the objective (blue dashed lines). A pattern corresponding to the proper phase modulation (different grey levels represent phase modulation from 0 to $2 \pi$ ) is addressed to the SLM. This phase modulation is imaged to the back focal plane of the objective and transformed to the desired intensity pattern at the sample plane. Note that in phase only modulation the intensity profile is characterized by speckle. 


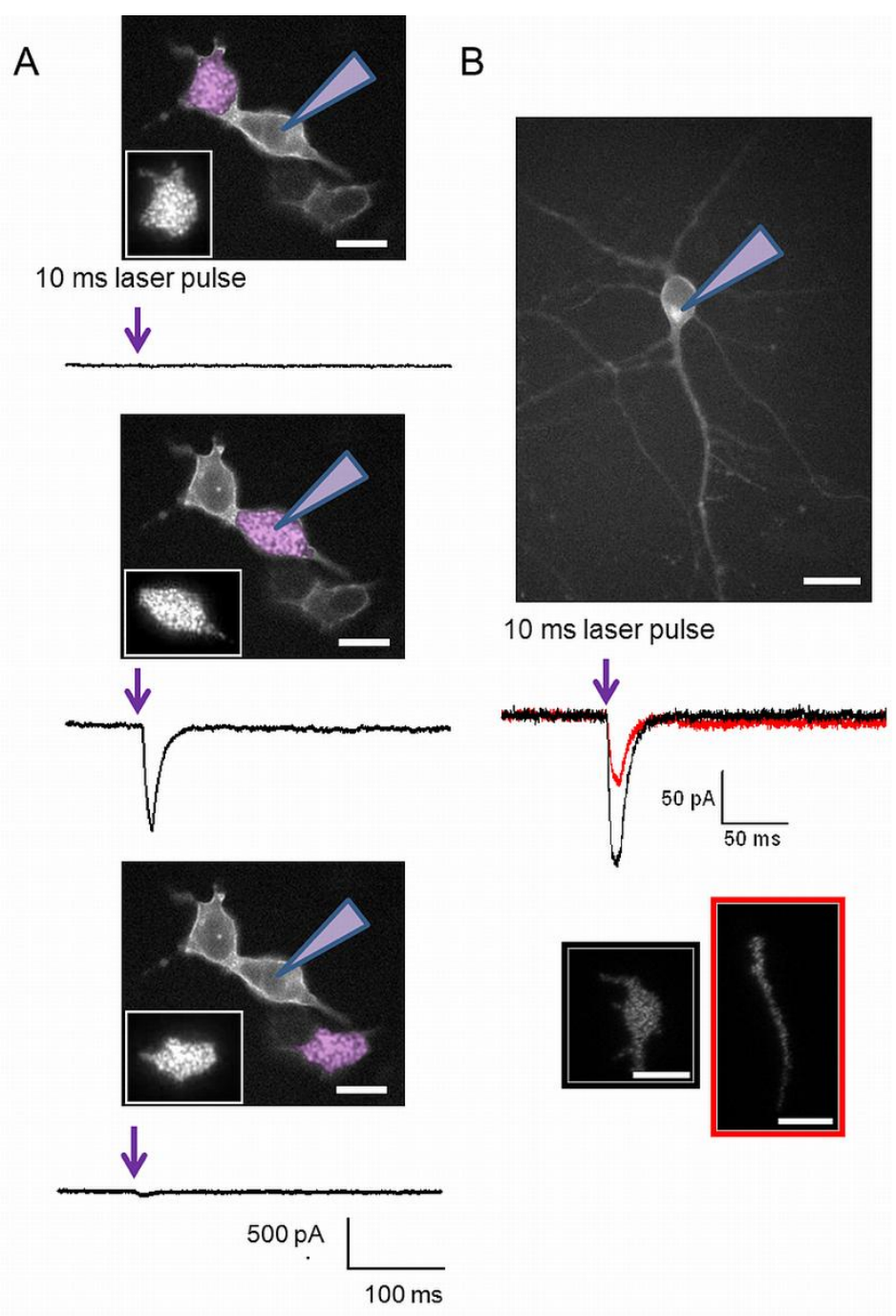

Figure 2 | Single-photon holographic excitation of ChR2

(A) Wide-field fluorescence images of HEK cells expressing GFP-tagged ChR2-H134R (top) are superimposed with holographic illumination patterns imaged on a thin layer of rhodamin-6G (in purple). The fluorescence excitation of each spot is shown separately (inset). Underneath each image is the corresponding electrophysiology recorded whole-cell current evoked by holographic photostimulation of ChR2. (B) Wide-field fluorescence image of a cultured neuron expressing GFP-tagged ChR2-H134R (top). Whole-cell currents (middle) evoked by holographic photostimulation of $\mathrm{ChR2}$ on the cell soma (black trace) or on the principal dendrite (red trace). The corresponding excitation patterns imaged on a rhodamin-6G thin film are shown in the black and red boxes. Excitation parameters: $\lambda=405 \mathrm{~nm}, 10 \mathrm{~ms}$ pulses, laser power $3.5 \mu \mathrm{W}$ at the exit of a 40x, 0.8 NA objective. Scale bars indicate $20 \mu \mathrm{m}$. Unpublished data, courtesy of $A$. Bègue, F. Anselmi and V. Emiliani (Wavefront-Engineering Microscopy Group, Neurophysiology and New Microscopies Laboratory). 

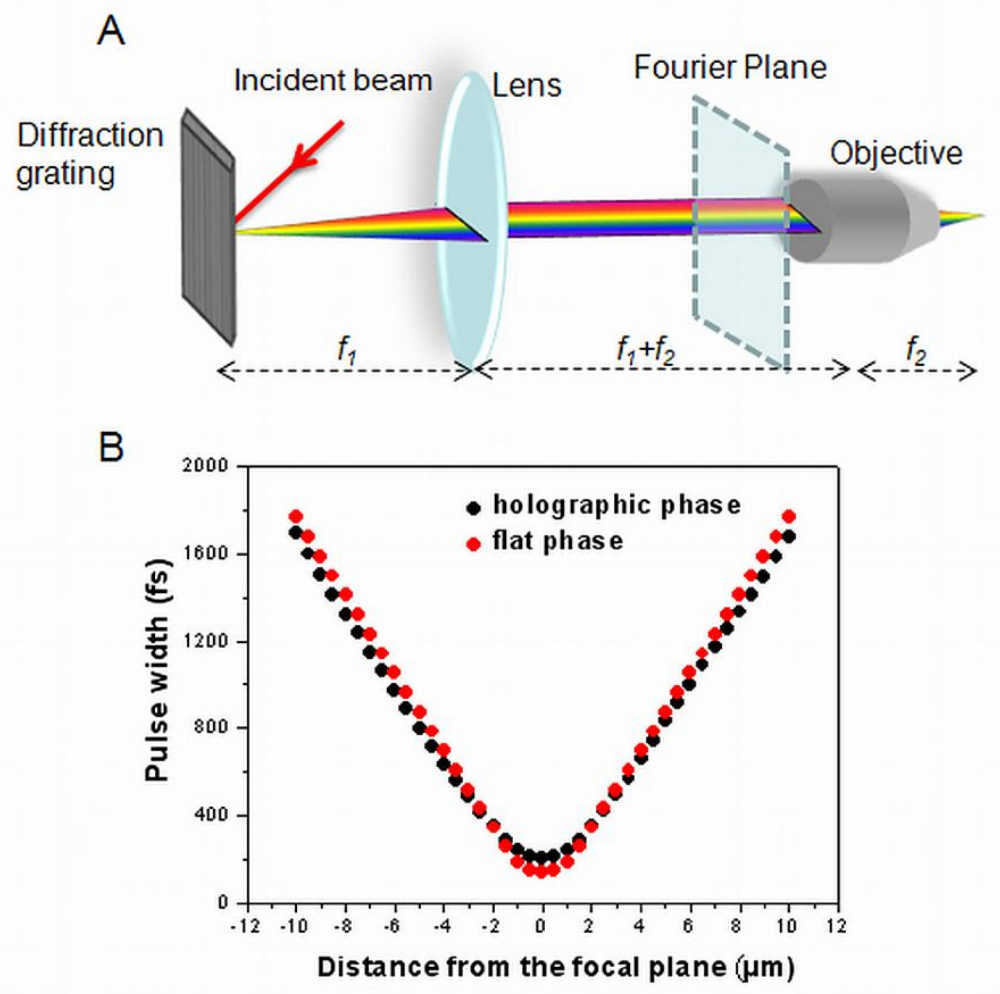

\section{Figure 3| Temporal focusing configuration}

(A) Illustration of the experimental configuration for TF. The diffraction grating is imaged onto the sample via a $4 f$ telescope formed by a lens and the microscope objective. The laser beam impinges on the grating at an angle such that the central frequency of the pulse is diffracted perpendicular to the grating, along the optical axis of the telescope. For large illumination areas on the sample and consequently on the grating as well, this configuration helps prevent illumination of the sample planes tilted relative to the microscope axis. (B) Plot of the pulse duration in a TF setup for different sample planes spaced up to $\pm 10 \mu \mathrm{m}$ from the focal plane of the objective. The pulse duration was calculated after simulating TF of $140-f$ s laser pulses centered at $780 \mathrm{~nm}(\Delta \lambda=12 \mathrm{~nm})$ impinging on an $830 \mathrm{I} / \mathrm{mm}$ groove density grating and a telescope comprised of a 500-mm lens and a 60x, 0.9 NA objective (see Papagiakoumou et al., 2008 for simulation details). Pulse width is plotted as a function of z-position for a flat-phase beam (e.g. a Gaussian beam; red dots) and a holographic beam (black dots). Pulse broadening to a few picoseconds at $10 \mu \mathrm{m}$ from the focal plane corresponds to an axial resolution of few microns ( $\sim 3 \mu \mathrm{m}$ for the Gaussian beam and 4-5 $\mu \mathrm{m}$ for the holographic beams for the setup described in Papagiakoumou et al., 2008). As evidenced by the calculation, holographic phase distorts recombination of different spectral components, resulting in larger pulse duration at the focal plane than for a flat-phase beam. This effect increases the excitation volume by 1-2 $\mu \mathrm{m}$ for a holographic beam in comparison to a Gaussian one in a TF setup. 

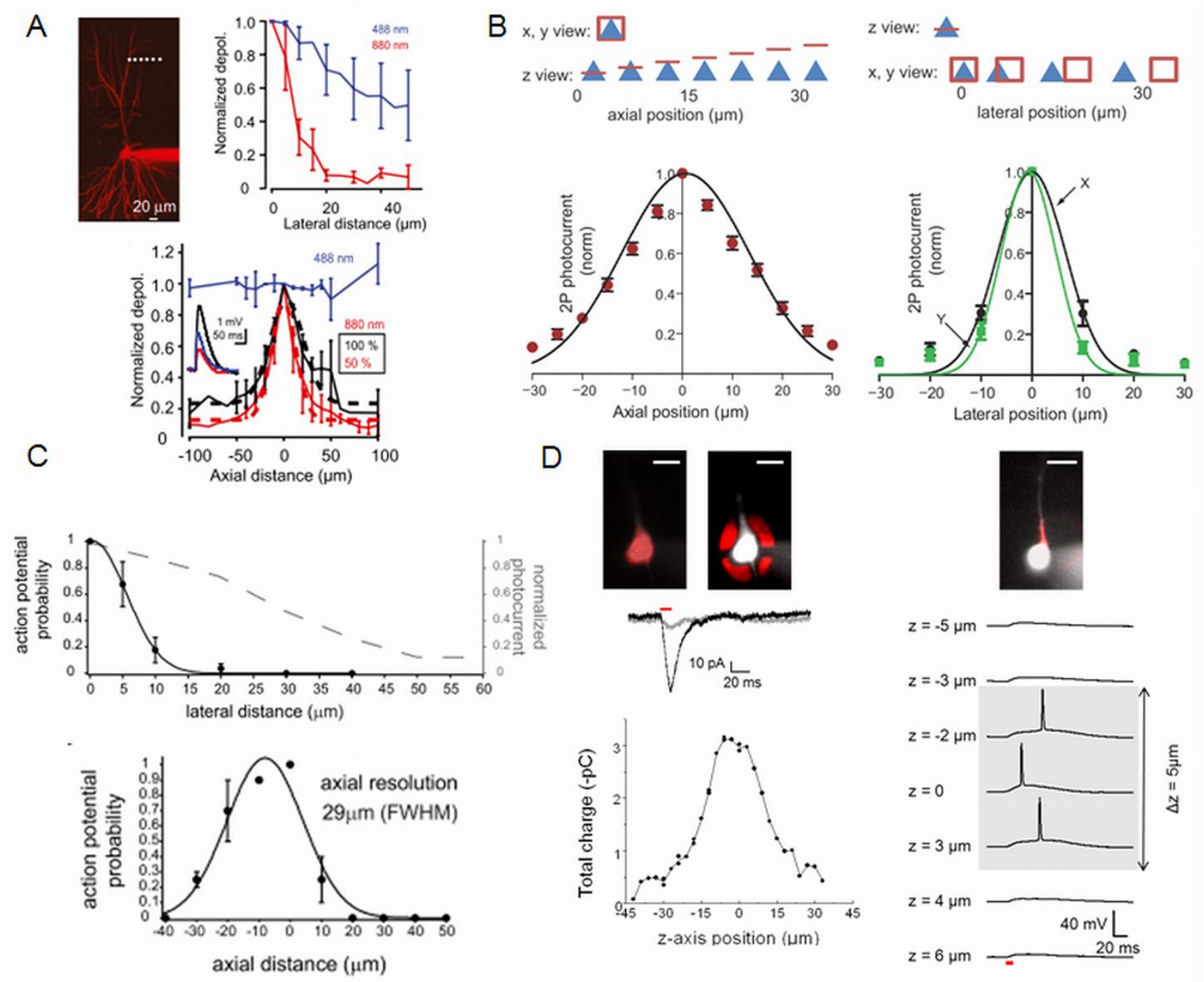

\section{Figure 4 | Axial resolution of 2PE approaches in optogenetics}

(A) Spatial resolution of ChR2 activation with temporally focused 5- $\mu$ m diameter spot scanned over a thin (1-2 $\mu \mathrm{m})$ apical dendrite of a CA1 pyramidal cell, adapted from Andrasfalvy et al., 2010. The image on the top left is a maximum intensity projection of a z-series through a pyramidal cell loaded with Alexa594, showing the lateral locations of the activation spots. The lateral and axial localization of responses induced by 488-nm light (blue curves) and temporally focused excitation (red or black curves) are shown on the top right and bottom panels. The axial responses were measured for two different power values, 130 mW (red) and 260 mW (black; dashed lines indicate Gaussian fit). Individual responses at the focal plane of the dendrite (colorcoded respectively) are shown on the inset. Note that the axial resolution improves with lower power. Nevertheless, the photoactivation volume of the $5-\mu \mathrm{m}$ spot extends in $\sim 35 \mu \mathrm{m}$ and 15 $\mu \mathrm{m}$ FWHM in the axial and lateral dimension respectively. (B) Axial (left) and lateral (right) resolution of scanning $2 \mathrm{PE}$ of $\mathrm{C} 1 \mathrm{~V} 1$ photocurrents from pyramidal cells in prefrontal cortex 
slices, adapted from Prakash et al., 2012. Top panels show schematics of the areas where a $\sim 1.5-\mu \mathrm{m}$ diameter spot (5.7 $\mu \mathrm{m}$ in the axial direction) was scanned relative to the center of the cell body. Blue triangles indicate a pyramidal neuron, and red boxes indicate a typical ROI (10x10 $\mu \mathrm{m}$ to $15 \times 15 \mu \mathrm{m})$. Graphs underneath show 2P photocurrent as a function of axial (left) and lateral (right; black curve for ' $x$ ' direction and green curve for ' $y$ ') distance of the ROI from the center of a pyramidal cell. The excitation wavelength was $\lambda=1040 \mathrm{~nm}$, the laser intensity was $20 \mathrm{~mW}$ at the sample plane and the Gaussian fit gives a FWHM of $29.5 \mu \mathrm{m}$ in the axial and 15-20 $\mu \mathrm{m}$ in the lateral dimension that roughly matches the size of the cell soma. (C) Spatial resolution of AP probability acquired by raster scanning of 1- $\mu \mathrm{m}$ diameter spot over a C1V1expressing pyramidal cell in somatosensory cortex, adapted from Packer et al., 2012. Lateral resolution of the photostimulation in relation to distance from soma is shown at the top ( $\lambda=1040 \mathrm{~nm}, 20 \mathrm{~mW}$ on sample). Although APs were produced in individual neurons with a lateral resolution of $6.5 \mu \mathrm{m}$, photocurrents are seen as far as $50 \mu \mathrm{m}$ away from the soma (grey dashed line). The lateral resolution for spiking is significantly higher due to the AP threshold. The axial resolution for AP probability generation is shown at the bottom. APs were produced in individual neurons with axial resolution of $29.5 \mu \mathrm{m}$. The corresponding photocurrent curve vs. axial distance from the soma is not shown. Note that the spatial resolution in this case is worse than in the data displayed in $\mathbf{B}$, although the excitation parameters are the same. This can be possibly attributed to the total scan time, which in Packer et al. is $73 \mathrm{~ms}$, while in Prakash et al. is $\sim 5$ ms. (D) Spatial resolution of the TF-GPC method, adapted from Papagiakoumou et al., 2010. To demonstrate lateral precision, we compared photocurrents excited by TF-GPC patterns of 4- $\mu \mathrm{m}$ axial resolution illuminating either the whole soma (shape) or the surrounding area (antishape) of a layer $\mathrm{V}$ pyramidal neuron in somatosensory cortical brain slices. The top right image shows wide-field fluorescence of a ChR2-YFP positive neuron filled with Alexa594 and superimposed excitation patterns (red) with shaped and anti-shaped profiles with corresponding photocurrents (black for shape, grey for antishape; 10 ms illumination duration, $0.24 \mathrm{~mW} / \mu^{2}$ at the sample plane). The bottom traces show the integrated photocurrent (area above the inward current in pico-coulombs) evoked by a $10-\mu \mathrm{m}$ diameter spot centered on the cell body when displaced along the $z$ axis $\left(0.30 \mathrm{~mW} / \mu \mathrm{m}^{2}\right)$. A FWHM of $27.5 \mu \mathrm{m}$ can be inferred for the axial precision of photocurrents induction. The method's precision was also measured by the probability of generating APs on a neuronal dendrite. The left image shows fluorescence of a ChR2-positive neuron filled with Alexa594 and the superimposed shaped excitation profile covering the apical dendrite (red), as well as the photo-depolarizations evoked by the excitation shape at different $\mathrm{z}$-axis positions (bottom panel; $10 \mathrm{~ms}$ illumination duration, $0.30 \mathrm{~mW} / \mathrm{\mu m}^{2}$ ). APs were generated only on a narrow band of $5 \mu \mathrm{m}$ around the focal plane. Scale bars indicate $20 \mu \mathrm{m}$ and the excitation wavelength was $\lambda=920 \mathrm{~nm}$. 

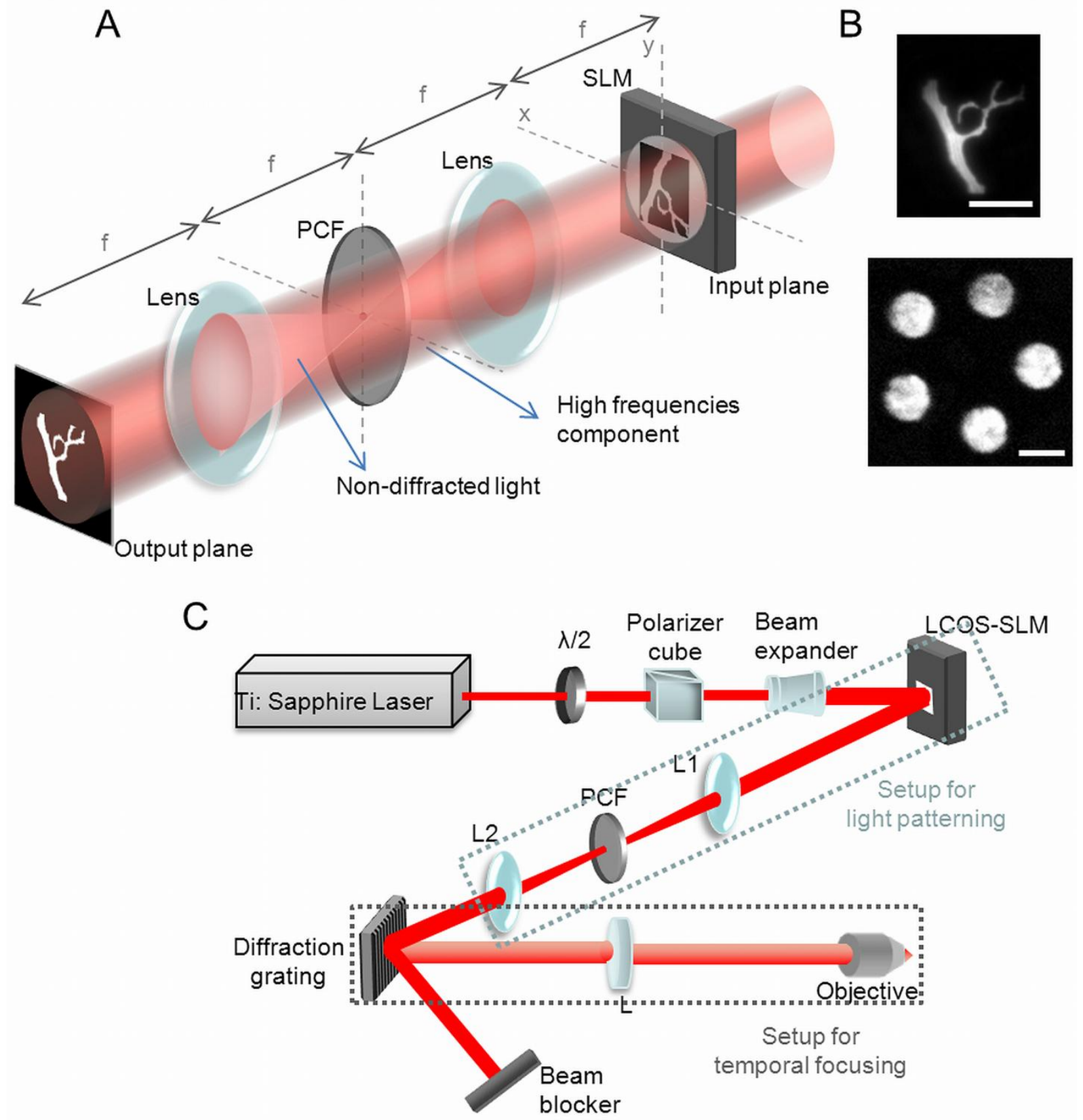

Figure 5| Generalized phase contrast

(A) Schematic layout of the $4 \mathrm{f}$ configuration for GPC. A binary phase map of the desired excitation pattern is sent on the SLM. A phase contrast filter (PCF) at the Fourier plane of the first lens introduces half-wave $(\pi)$ phase retardance between the component not diffracted by the SLM and the high-frequency component (signal). Interference of the two light components 
at the output plane gives an intensity pattern according to the phase modulation at the input plane (constructive interference at the areas that were addressed with phase $\pi$ and destructive interference at the areas addressed with phase 0). (B) Examples of GPC-generated patterns with $2 \mathrm{PE}$. The pattern at the top mimics the dendrite of a Purkinje cell, while the one on the bottom shows simultaneous multi-spot excitation. $2 P E$ is visualized on thin rhodamine-6G films. GPC patterns, unlike $\mathrm{DH}$, are free of speckle. (C) Schematic layout of a setup for combining GPC with TF. Notably, the lenses used to form the $4 \mathrm{f}$ system for GPC do not need to have equal focal lengths. In this way the excitation field can be adjusted to the needs of the experiment.

\section{Graphical abstract}

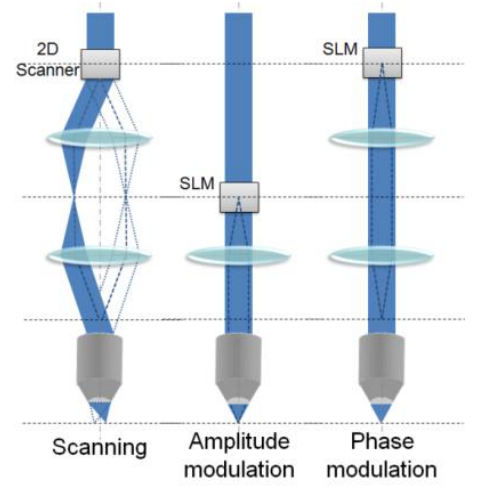

Advances in molecular engineering and the bloom of optogenetics facilitated the realization of studies towards the understanding of neural circuits function. To this direction great effort has been made to develop also illumination tools that provide better specificity than standard widefield illumination. Here optical methods that have been used so far in optogenetics are reviewed and the challenges that need to satisfy are discussed. 